\title{
Faults identification and corrective actions in rotating machinery at rated speed
}

\author{
Nicolò Bachschmid and Paolo Pennacchi* \\ Dipartimento di Meccanica, Politecnico di Milano, Via La Masa 34, I-20156 Milano, Italy
}

\begin{abstract}
Malfunction identification in rotor systems by means of a model based approach in the frequency domain during long lasting speed transients (coast-down procedures in large turbo-generators), where a huge amount of vibration data at different rotating speeds is usually collected, has proved to be very effective. This paper explores the possibility to adapt this method to the situation when the vibration data are available at one rotating speed only, which in real machines is generally the normal operating speed. It results that single speed fault identification can be successful, but does not allow to discriminate between different malfunctions that generate similar symptoms. Neverthless the identification results can be used to define corrective balancing masses.
\end{abstract}

Keywords: Rotor dynamics, diagnostics, identification

\section{Introduction}

The authors have developed a malfunction identification procedure for rotor systems based on the model of the system (shafts, bearings and supporting structure), on suitable models of the different malfunctions and on a least square approach in the frequency domain. This procedure analyses the vibration data usually collected by monitoring systems in correspondence of the bearings only, at different rotating speeds selected from a long lasting coast-down transient, which is considered as a sequence of steady state operating conditions. The procedure has been applied to several turbo-generators (steam and gas) of power stations, and proved to be effective and robust, provided that the machine exhibits linear behavior and has time invariant characteristics. Generally turbo-generators composed of steam turbines and generators fulfill this condition; on the contrary turbo-generators composed of gas turbines and generators exhibit behaviors which are scarcely repetitive, which do not fulfill this condition.

A diagnostic system should be able to predict an impending fault also during normal operating conditions at rated speed. This is obviously a much more difficult task, because the available information at one speed only are much less. Some single speed identification procedures in the time domain $[3,5]$ seem to be successful, but have never been used with real machine vibration data.

The aim of this research is therefore to check if and to which extent the model based approach in the frequency domain is able to fully identify the impending fault (i.e. the type, the position along the rotor and the severity), by using the data of one rotational operating speed only, both in case of test-rigs and of real machines, and possibly to suggest corrective actions. Advanced signal analysis techniques allow to define completely a symptom, which can be used in the well-known rule-based symptom-fault identification approach, which does not allow the severity and location of the impending fault to be identified.

The approach uses the finite beam element model of the shaft, bearings are represented as usual by stiffness and damping coefficients, the supporting structure is represented by mass, spring and damper systems, or, when available, by a modal model of the structure [4]. In the identification procedure, malfunctions like instabilities or looseness of

*Corresponding author. Tel.: +3922399 8440; Fax: +392 2399 8492; E-mail: paolo.pennacchi@ polimi.it. 

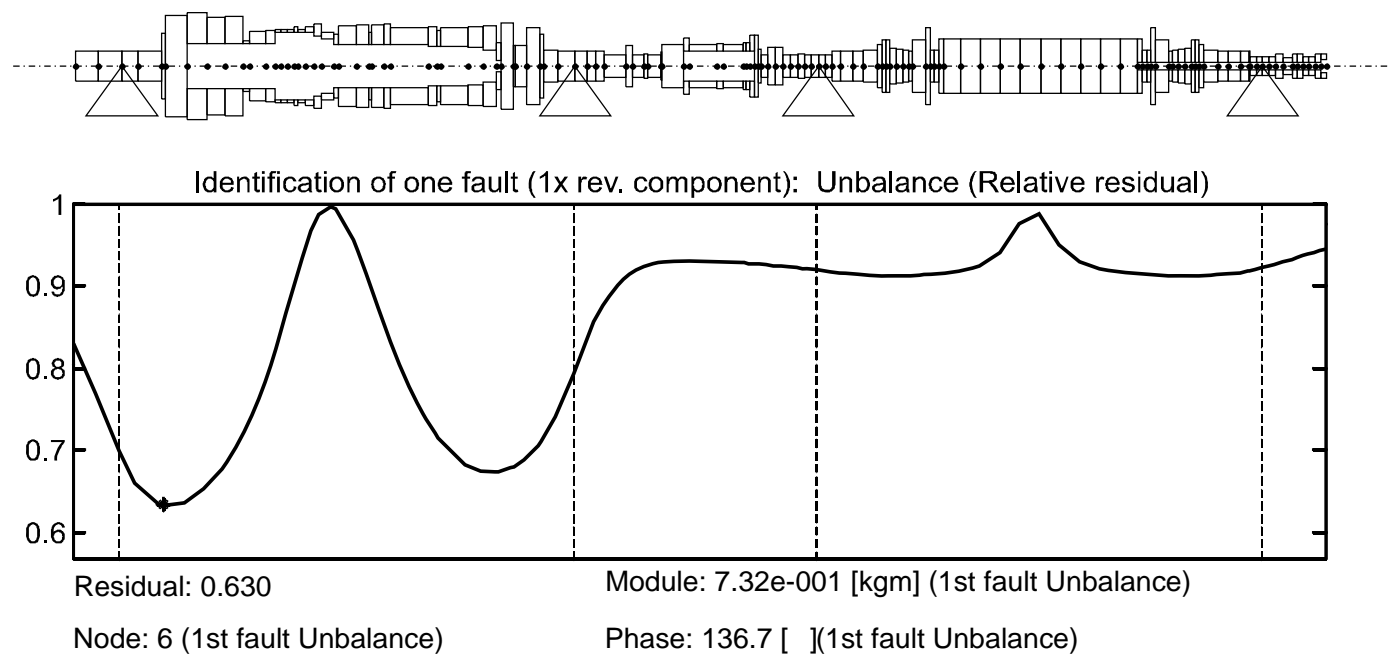

Fig. 1. Application 1: gas turbine and generator, $125 \mathrm{MW}$ - Identification results using the data corresponding to one rotating speed only (actual fault: unbalance, node 6 , module $0.373 \mathrm{kgm}$ phase $157.5^{\circ}$ ).

parts are not taken into consideration. Also single faults only are considered, the possibility of multiple coexisting faults is not accounted for.

All the considered malfunctions are modeled by means of equivalent exciting forces. These are generally applied on one location only along the rotor, and are often composed by one rotating force only (e.g. unbalance) or by two equal and opposite bending moments applied to the nodes of one element (e.g. local bow due to a rub, transverse crack, coupling misalignment), or to the extremity nodes of a set of elements representing a huge part of the rotor (in case of thermal bow or distributed axial asymmetry). Therefore malfunction identification is reduced to just one equivalent external force (or couple of bending moments) identification: one or two complex unknowns are to be identified, and the measured data are generally at least four complex vibration vectors (at least two bearings and two directions of measurement) at each rotating speed. The number of known quantities is higher than the number of unknowns, therefore the least square approach can be used also in case that only one rotating speed is taken in consideration (the normal operating speed), instead of a set of $n$ different rotating speeds chosen during the long lasting speed transient.

The data which are used for the identification procedure at one rotating speed only, are much "poorer" in information content than the huge amount of data which are at disposal during the speed transients.

Especially some important frequency dependent features of the equivalent forces are completely lost: the information that the unbalance depends on the square of the speed, and that the local bow and similar malfunctions are represented by a constant amplitude force system, cannot be exploited in the identification procedure at one rotating speed only.

In order to explore the capability of the method in malfunction identification, despite the described shortcomings, several different conditions in test-rigs and in real machines are taken into consideration. Another important feature of an identification procedure is also its capacity to distinguish between different malfunctions which exhibit similar symptoms. The investigation will show also which are the limits of the procedure in this sense. Finally, an example shows how the identification results can be used to define a set of balancing masses able to reduce vibration levels at rated speed.

\section{Description of the method}

Assuming a finite beam element model for the rotor, the effect of a fault can be simulated in the frequency domain by applying to the rotor different sets of equivalent forces. The problem of the identification of the position and 

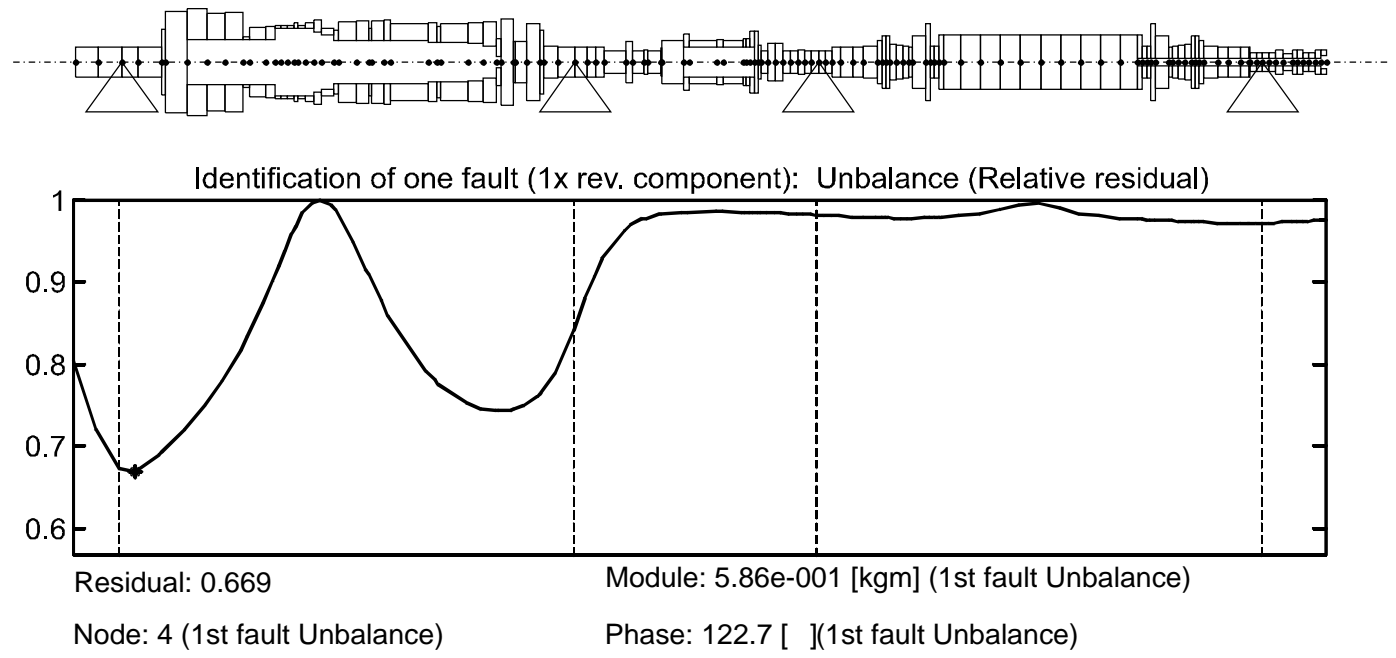

Fig. 2. Application 1: gas turbine and generator, $125 \mathrm{MW}$ - Identification results using the data corresponding to all the available rotating speeds (actual fault: unbalance, node 6 , module $0.373 \mathrm{kgm}$ phase $157.5^{\circ}$ ).

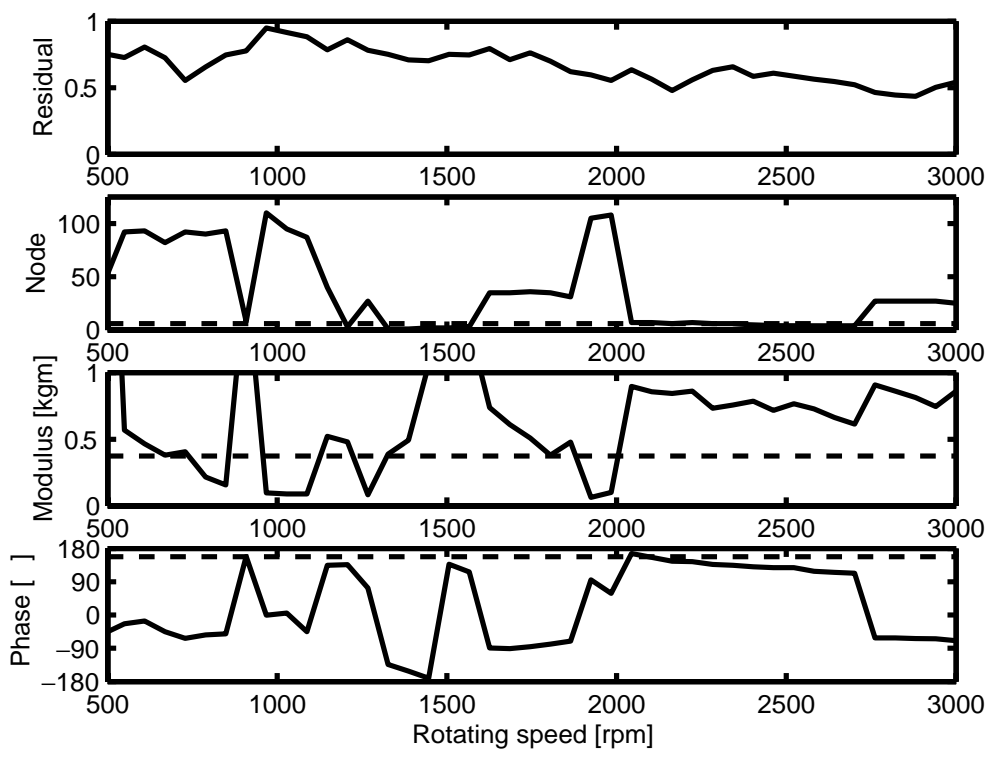

Fig. 3. Application 1: residual, node, modulus and phase identified as functions of the rotating speed.

magnitude of the fault is then reduced to an external force identification procedure, described in [2]. The final equations are recalled below.

The difference, between the measured vibration of rotor system that has a fault and the reference case, represents the vibrational behavior due to the fault, which is called sometimes "additional vibrations". These vibrations are then used in the identification procedure. By applying the harmonic balance criteria in the frequency domain, the following equations are obtained for each harmonic component, in which the force vector, $\mathbf{F}_{f_{n}}$, has to be identified:

$$
\left[-(n \Omega)^{2} \mathbf{M}+i n \Omega \mathbf{C}+\mathbf{K}\right] \mathbf{X}_{n}=\mathbf{F}_{f_{n}}
$$

Since linearity in the system is considered, the effect of $m$ faults developing simultaneously can be considered by means of the superposition of the effects for each harmonic component: 
Additional vibrations, beaning \#1
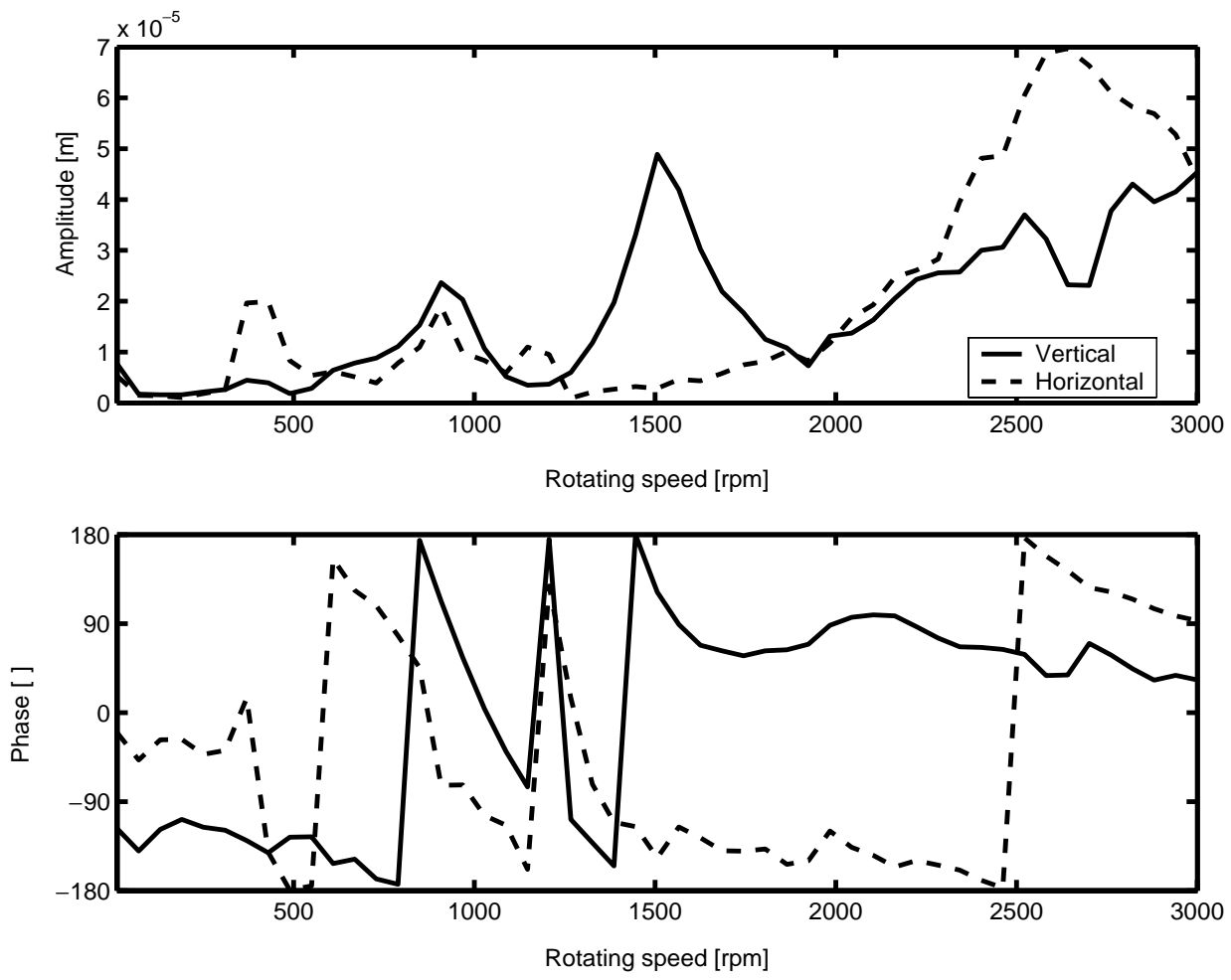

Fig. 4. Application 1: additional vibrations due to the unbalace on node 6, modulus $0.373 \mathrm{kgm}$ phase 157.5 measured in bearing \#1.

$$
\mathbf{F}_{f_{n}}=\sum_{k=1}^{m} \mathbf{F}_{f_{n}}^{(k)}
$$

Moreover, the $k$-th fault acts on few degrees of freedom (d.o.f.s) of the system, therefore the vector $\mathbf{F}{ }_{f_{n}}^{(k)}$ is not a full-element vector which is convenient to be represented by means of:

$$
\mathbf{F}_{f_{n}}^{(k)}=\left\{\mathbf{F}_{L}^{(k)}\right\} \bar{A}^{(k)}(\Omega)
$$

where $\left\{\mathbf{F}_{L}^{(k)}\right\}$ is the localization vector which has all null-elements except for the d.o.f.s to which the forcing system is applied, and $\bar{A}^{(k)}(\Omega)$ is a complex number representing the amplitude and the phase of the equivalent force of the fault.

Then, introducing the admittance matrix of the system, Eq. (1) becomes:

$$
[\mathbf{E}(n \Omega)] \mathbf{X}_{n}=\mathbf{F}_{f_{n}}(\Omega)
$$

Normally, only a developing fault is going to be identified and the equivalent force fault identification problem in Eq. (4) is over-determined since the number of the observation (the measured vibrations) are grater than the number of the parameters of the fault (modulus and phase) that have to be identified. Least square identification is used in order to evaluate the module, the phase and a residual of a particular fault type in this way.

The equivalent force system is applied in each node of the rotor model. The effect on the measured d.o.f.s $\left[\hat{\mathbf{X}}_{\mathbf{B}_{n}}\right.$ ] due to unitary force systems applied in the first node on the model is calculated. This is done by inverting the matrix $[\mathbf{E}(n \Omega)]$ :

$$
\mathbf{X}_{n}=[\mathbf{E}(n \Omega)]^{-1} \mathbf{F}_{f_{n}}(\Omega)=[\mathbf{H}(n \Omega)] \mathbf{F}_{f_{n}}(\Omega)
$$


Additional vibrations, beaning \#2
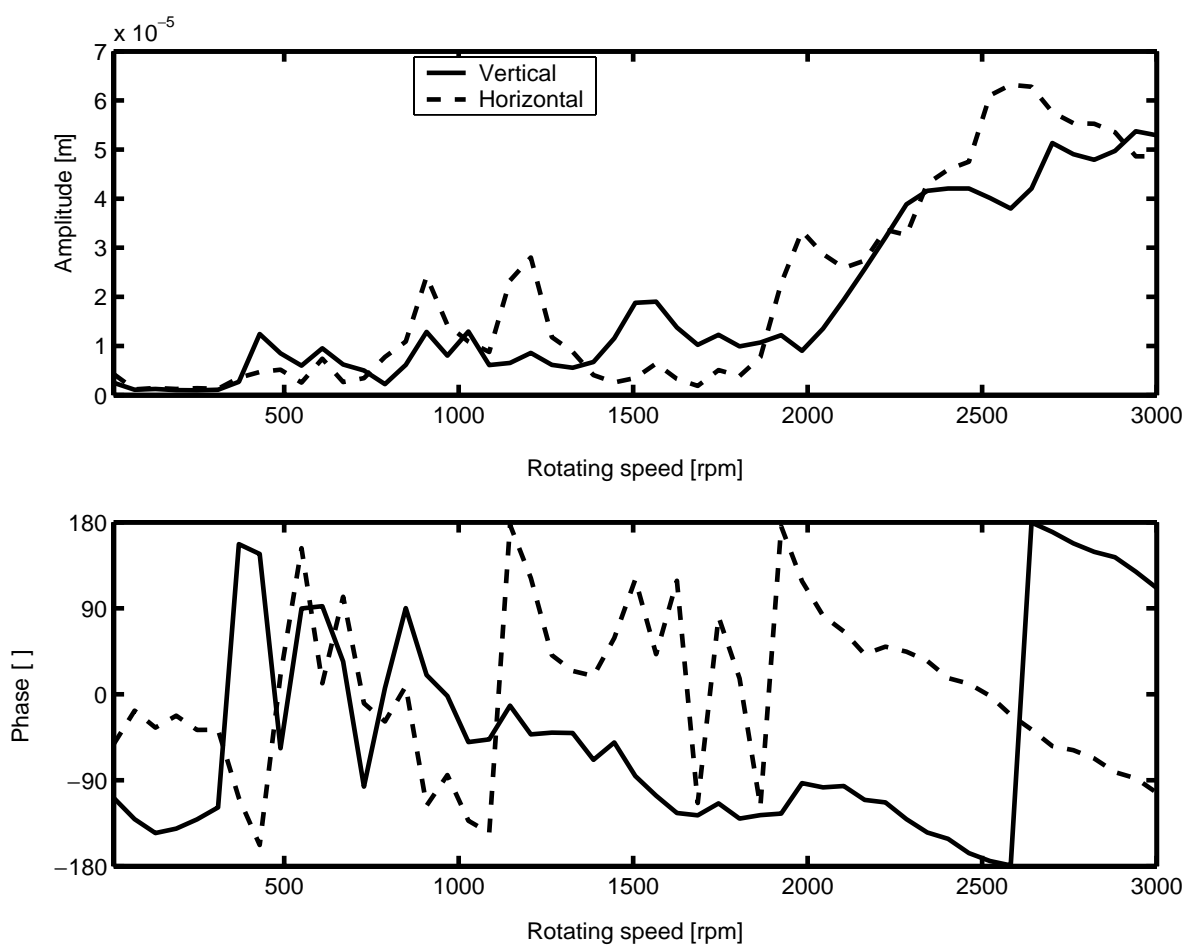

Fig. 5. Application 1: additional vibrations due to the unbalance on node 6, modulus $0.373 \mathrm{kgm}$ phase 157.9 measured in bearing \#2.

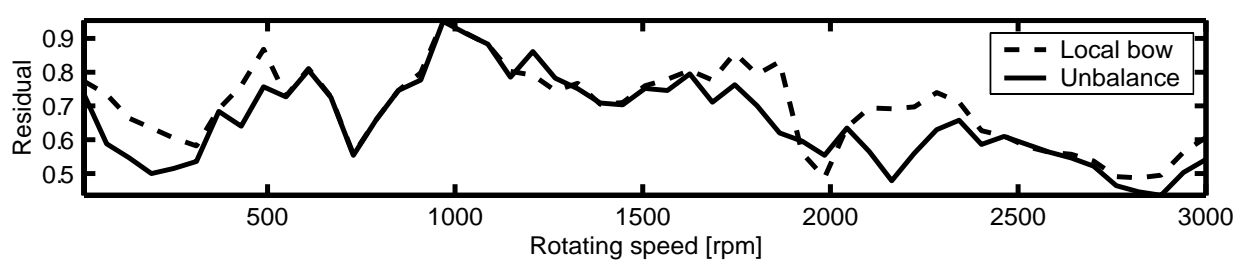

Fig. 6. Application 1: comparison between the residuals of two different fault types.

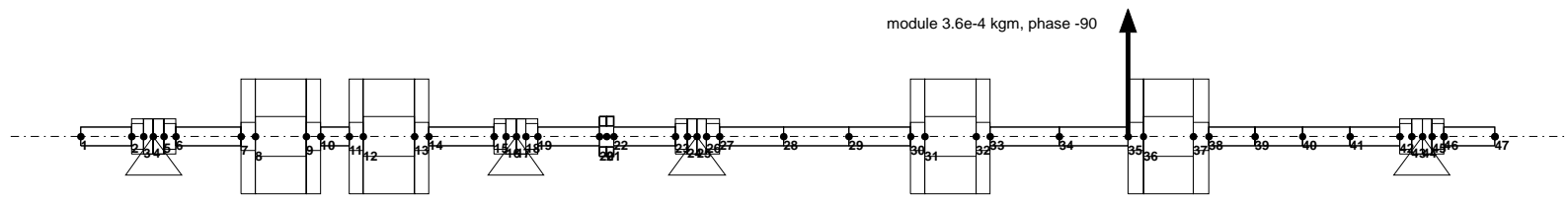

Fig. 7. Application 2: Modiarot test-rug.

Then, the vibrations of the d.o.f.s, which are measured, are separated from all the d.o.f.s of the system by considering only the rows of $[\mathbf{H}(n \Omega)]$ corresponding to the measured d.o.f.s.

It results:

$$
\left[\hat{\mathbf{X}}_{B_{n}}\right]=\left.[\mathbf{H}(n \Omega)]\right|_{\text {mesured }}\left[\mathbf{F}_{L}^{(1)} \ldots \mathbf{F}_{L}^{(m)}\right]
$$

Now the array of the complex values $\bar{A}^{(i)}$ (i.e. the modules and phases) of the equivalent force systems applied in the first possible location on the rotor that fits best the experimental data $\mathbf{X}_{B m_{n}}$, have to be estimated. The problem 

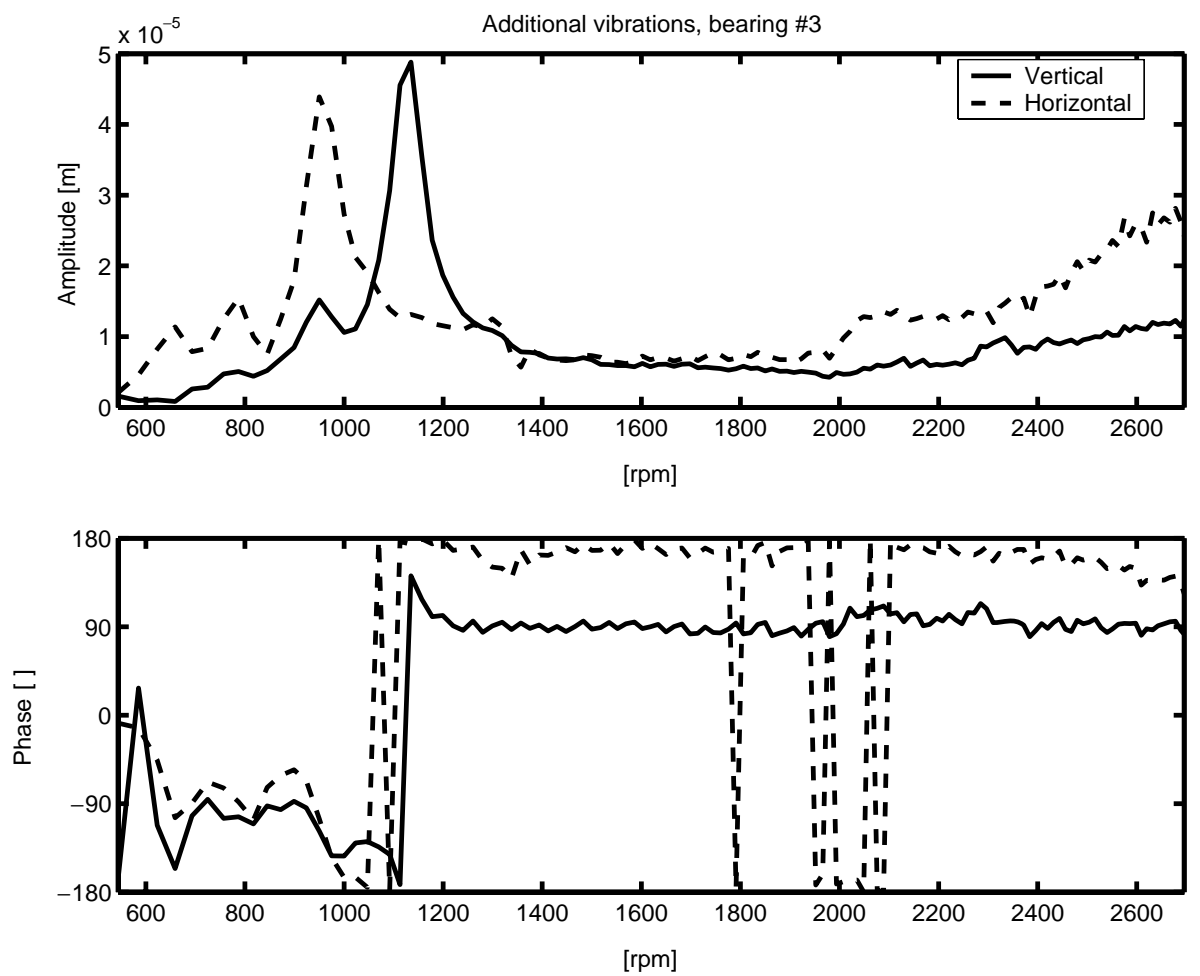

Fig. 8. Application 2: additional vibrations due to the unbalance on node 35 , modulus $3.6 \mathrm{e}-4 \mathrm{kgm}$ phase -99 measured in bearing $\# 3$.

is equivalent to minimize the $\ell^{2}$-norm:

$$
\min \left\|\left[\hat{\mathbf{X}}_{B_{n}}\right]\left\{\begin{array}{l}
\bar{A}^{(1)} \\
\vdots \\
\bar{A}^{(m)}
\end{array}\right\}-\mathbf{X}_{B m_{n}}\right\|
$$

whose general solution is given by solving the system:

$$
\left[\hat{\mathbf{X}}_{B_{n}}\right] \mathbf{A}^{(1)}-\mathbf{X}_{B m_{n}}=0
$$

by means of the pseudo-inverse calculation:

$$
\mathbf{A}^{(1)}=\left(\left[\hat{\mathbf{X}}_{B_{n}}\right]^{T}\left[\hat{\mathbf{X}}_{B_{n}}\right]\right)^{-1}\left[\hat{\mathbf{X}}_{B_{n}}\right]^{T} \mathbf{X}_{B m_{n}}
$$

The modules and the phases of the complex values in the $m$ rows of $\mathbf{A}^{(1)}$ are the identified faults in the first rotor node. Finally the residual in the first rotor node is determined, first obtaining the calculated response due to the identified fault in the first node:

$$
\mathbf{X}_{B n}=\left[\hat{\mathbf{X}}_{B_{n}}\right] \mathbf{A}^{(1)}
$$

and then normalizing it:

$$
\delta_{r_{n}}^{(1)}=\left(\frac{\left[\mathbf{X}_{B_{n}}-\mathbf{X}_{B m_{n}}\right]^{* \mathrm{~T}}\left[\mathbf{X}_{B_{n}}-\mathbf{X}_{B m_{n}}\right]}{\mathbf{X}_{B m_{n}}^{* \mathrm{~T}} \mathbf{X}_{B m_{n}}}\right)^{1 / 2}
$$

The procedure is then iterated for all the $n_{r}$ nodes of the rotor. If only one fault is considered, a set in $\mathbb{R}$ of relative residuals given by Eq. (11), ordered by the node number of the fault, is obtained: 
Additional vibrations, bearing \#4
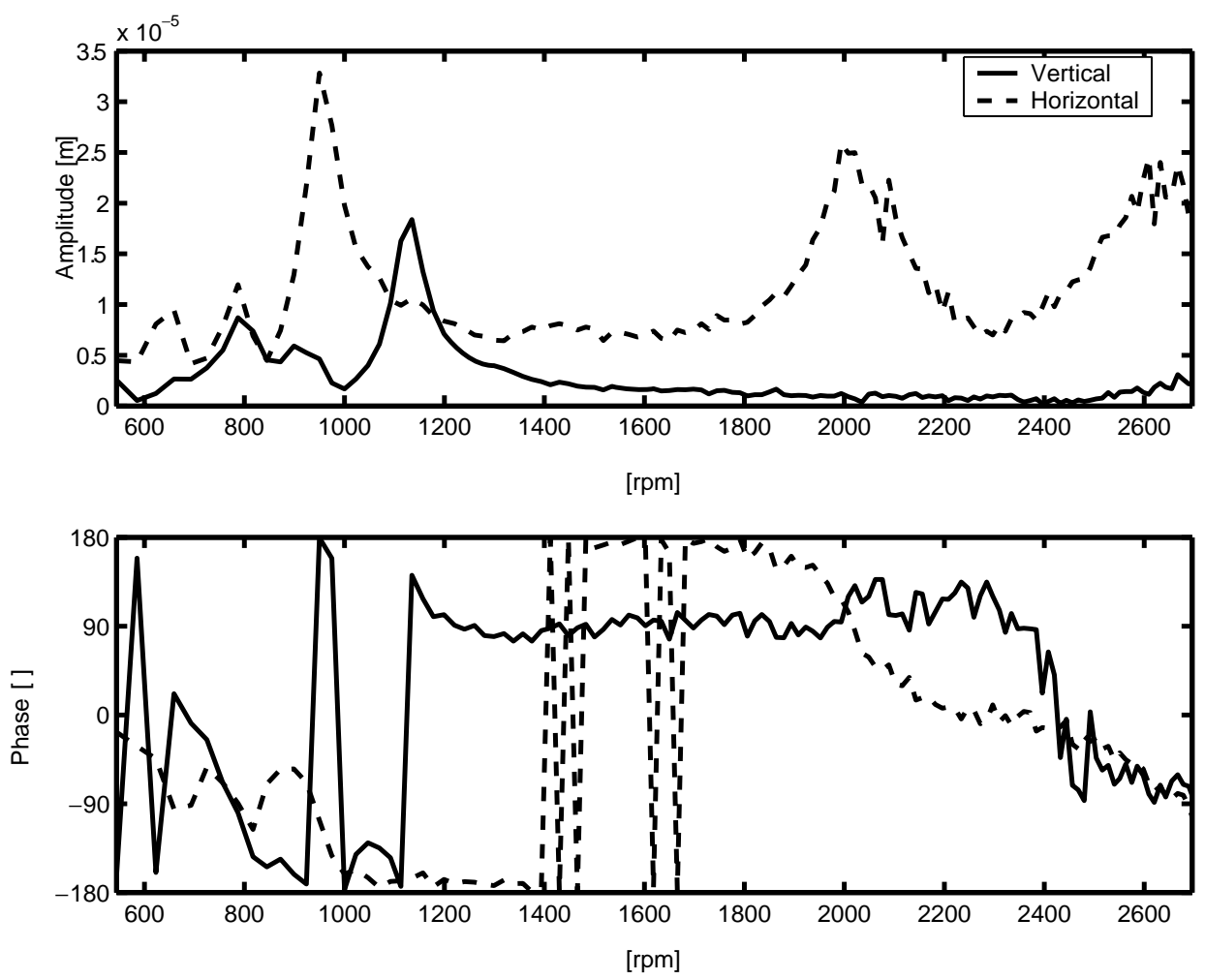

Fig. 9. Applications 2: additional vibrations due to the unbalance on node 35 , modulus $3.6 \mathrm{e}-4 \mathrm{kgm}$ phase $-9 \oplus$ measured in bearing $\# 4$.

$$
\delta_{r_{n}}=\left(\delta_{r_{n}}^{(1)}, \ldots, \delta_{r_{n}}^{\left(n_{r}\right)}\right)
$$

The $s$-th node location that corresponds to the minimum component of the vector in Eq. (12) indicates the most probable location of the fault, whose estimation is given by the corresponding value of Eq. (9).

\section{Application to test-rigs and industrial machines}

Several different applications are examined, and the results are discussed trying to find reasons for successful and unsuccessful identifications.

\subsection{Application 1: 125 MW gas turbo-generator affected by known unbalance}

Figure 1 shows the results of a quite successful identification at one rotating speed only ( $2283 \mathrm{rpm})$, which is not rated speed, compared to the result of the identification procedure which uses the data collected at all the available rotating speeds (Fig. 2): the position is identified with good accuracy, the amount is overestimated when using one speed only. The value of the residual is low in both cases indicating good quality in the identification procedure. But if another value of the rotating speed is used, the identification can be also completely unsuccessful.

In order to have an overview of the identification procedure success at different rotating speeds the identified quantities (position, amount and phase) are plotted as functions of the rotating speed.

Figure 3 shows the value of the residual, the node where the unbalance has been identified, its module and phase.

These values are compared to the true values (dotted lines). As can be seen, only in the speed range 2050$2700 \mathrm{rpm}$ the identification leads to correct results. This is probably due to the irregular behavior shown in Figs 4 

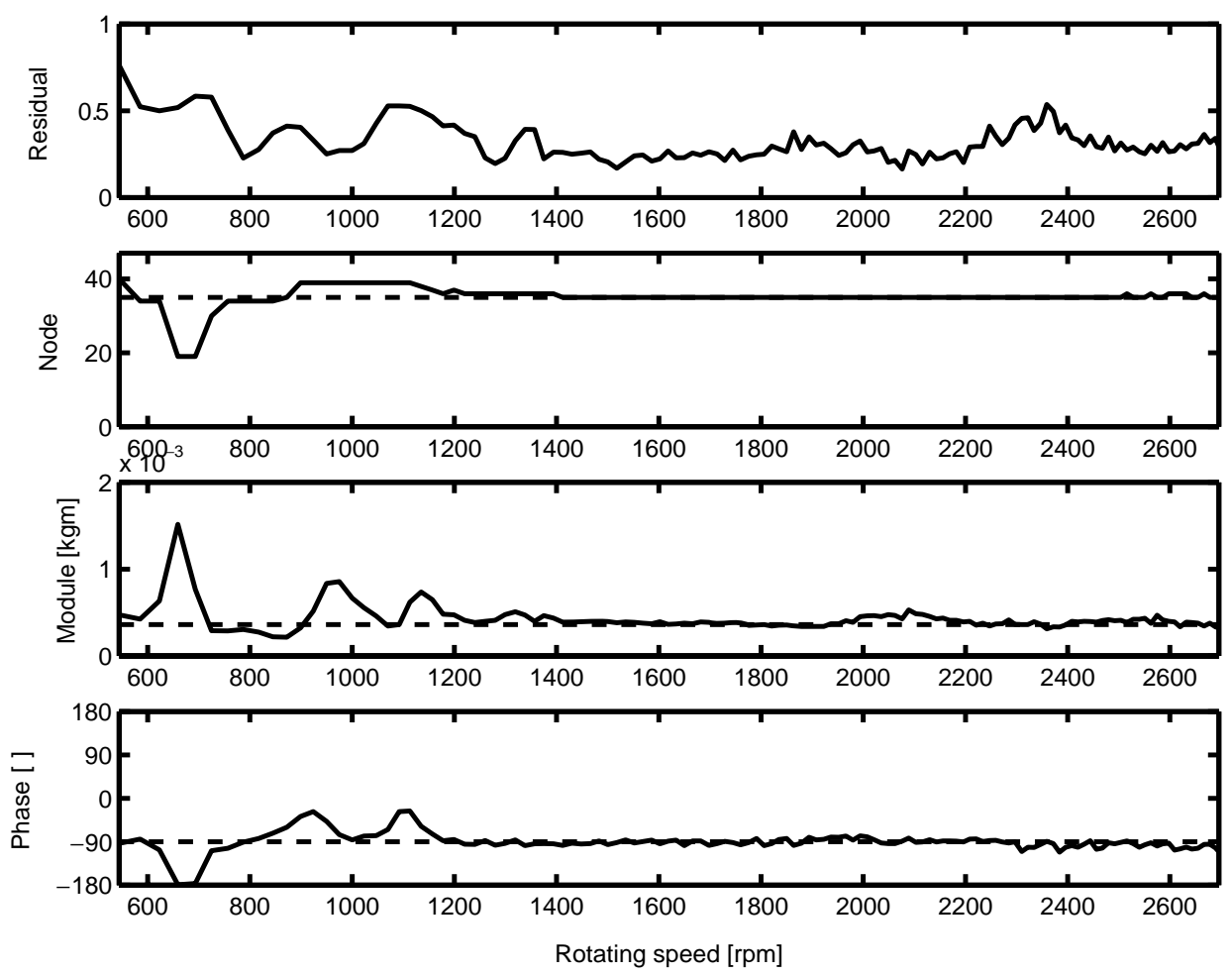

Fig. 10. Application 2: residual, node, modulus and phase identified as functions of the rotating speed.

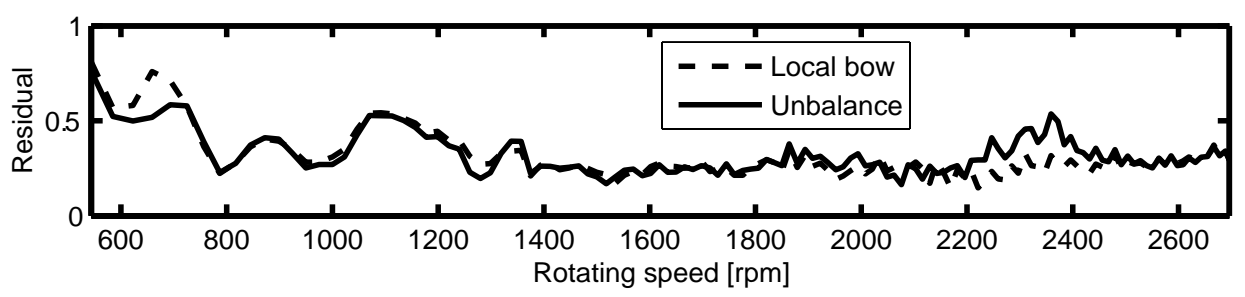

Fig. 11. Application 2: comparison between the residuals of two different fault types.

and 5, which represent additional vibrations obtained by subtracting from "faulty" vibrations the reference "healthy" vibrations. This irregular behavior is apparently due to a scarcely repetitive behavior of this kind of machine in different coast-down transients, and cannot be represented by linear and time invariant models.

Figure 6 compares the values of the residuals related to the unbalance to those related to a local bow which have similar symptoms (both generating only 1xrev. vibration components). It shows that when one speed only is considered, it is impossible to distinguish between malfunctions which generate similar symptoms by analyzing the values of the residuals. In these cases, only the likelihood of the identified positions for unbalance or local bow could help in distinguishing the impending fault: locations in which local bows are unlikely to develop or unbalances can not be applied might help to discard one or the other malfunction.

\subsection{Application 2: Modiarot test-rig with unbalance}

The Modiarot test-rig developed at the Dept. of Mechanics of Politecnico di Milano is composed by 2 shafts on 4 oil-film bearings on a flexible steel foundation. The shaft diameter is $25 \mathrm{~mm}$ and the total length of the two rigidly coupled shafts is about $2 \mathrm{~m}$ (exactly $725 \mathrm{~mm}$ the short and $1225 \mathrm{~mm}$ the long shaft), the total mass is a little less 

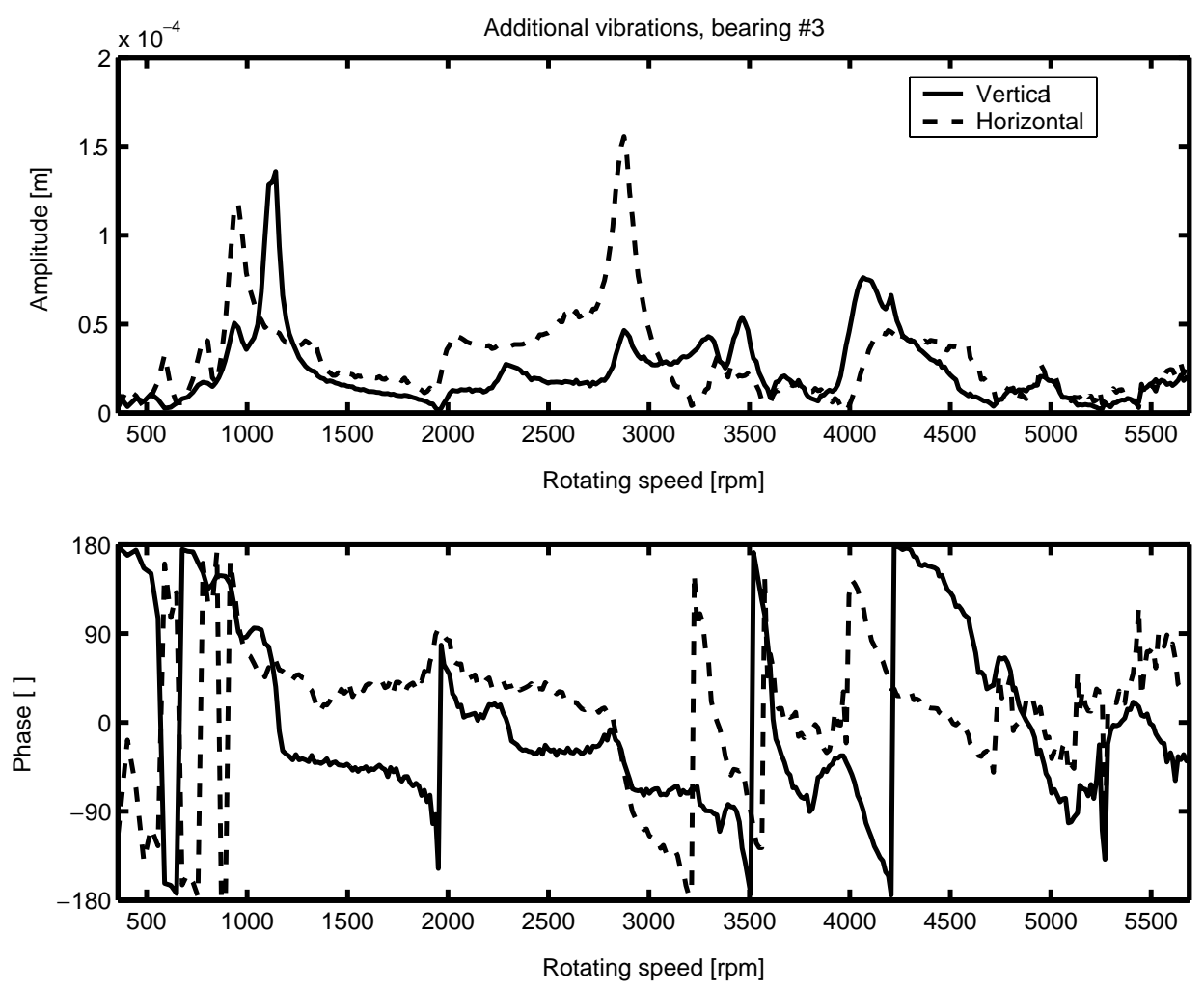

Fig. 12. Application 3: additional vibrations due to the coupling angular misalignment, modulus $5.45 \mathrm{mrad}$ phase -129 measured in bearing \#3.

than $100 \mathrm{~kg}$. Two shrink-fitted disks are mounted on each shaft. Two proximity probes in each bearing measure the relative shaft displacements, or the journal orbits; two accelerometers on each bearing housing measure its absolute vibrations.

The model of the shaft and the applied unbalance are shown in Fig. 7.

The mathematical model includes a modal representation of the flexible foundation. Figures 8 and 9 show the additional vibrations: several resonances of shaft and of supporting structure occur in the lower speed range (600-1400 rpm), apparently excited by the applied unbalance only.

Figure 10 shows the results of the identification procedure for single rotating speeds: in the higher speed range (1400-2700 rpm) the identification has success. Position, modulus and phase are identified with good accuracy, the low value of the residual indicates good quality of the identification procedure.

But in the low speed range where resonances occur the identification results become increasingly wrong. This confirms that where high dynamic responses of the mechanical system are measured, with rapid changes of amplitudes and phases, the model based identification fails because the model is not able to follow exactly the experimental behavior.

Figure 11 shows again that unbalance cannot be distinguished by local bow or other causes which generate only 1 xrev. components by using only a single measurement speed.

\subsection{Application 3: Modiarot test-rig with coupling misalignment}

In the same test-rig a coupling angular misalignment of $5.45 \mathrm{mrad}$ with an angular phase of $-120^{\circ}$ has been introduced. The obtained additional vibrations in bearings 3 and 4 are represented in Figs 12 and 13, where a rather "rich" dynamic behavior is shown. In this application the test-rig was operated up to its maximum speed of 5700 rpm. 

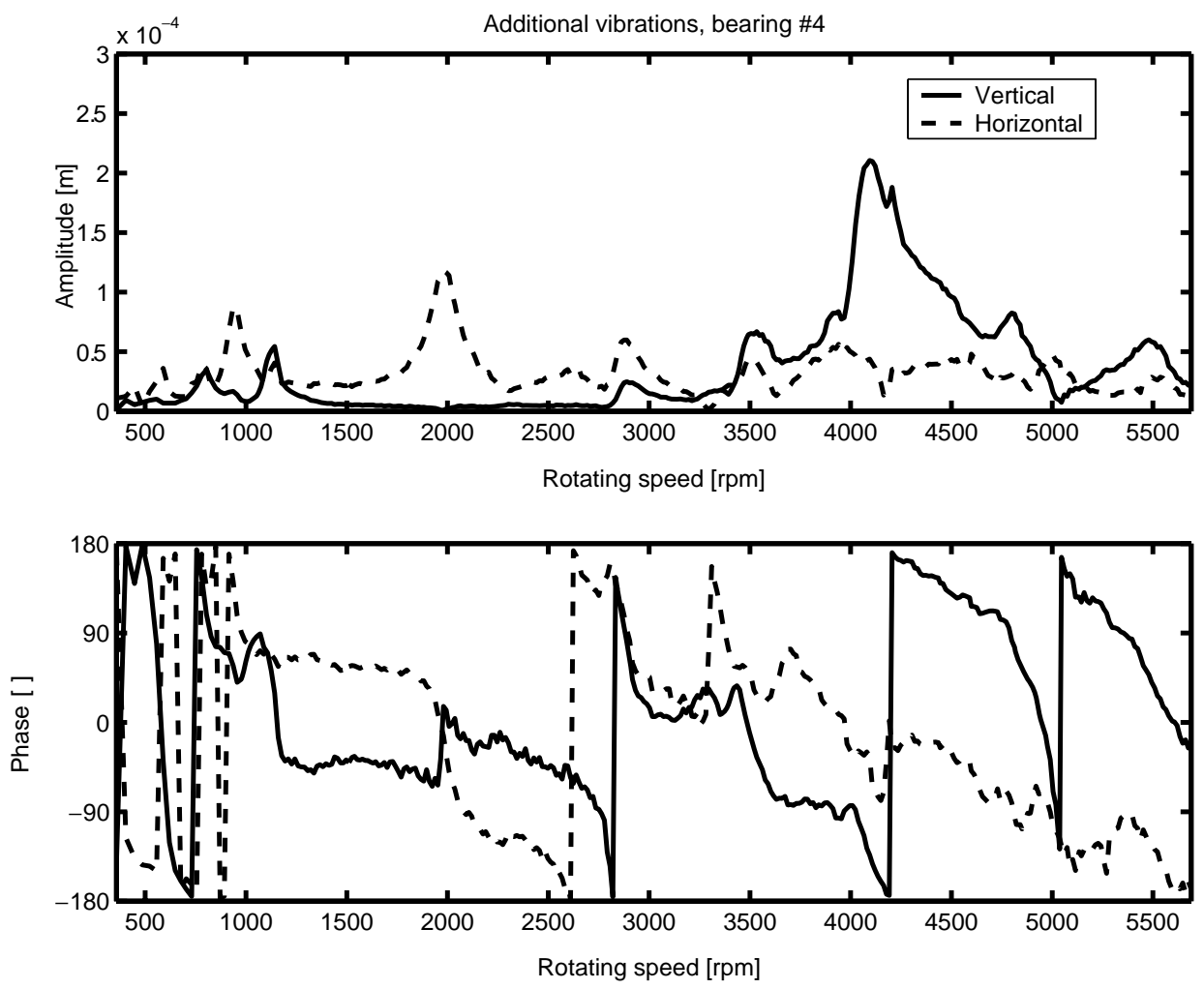

Fig. 13. Application 3: additional vibrations due to the coupling angular misalignment, modulus $5.45 \mathrm{mrad}$ phase -129 measured in bearing \#4.

Figure 15, in which the residuals of angular misalignment are compared to those of unbalance, shows as usual that single speed identification is unable to distinguish between malfunctions with similar symptoms.

Considering instead a selection of rotating speeds obtained by discarding speed ranges in which resonances occur, the angular misalignment is not only identified with high accuracy, but, due to the low value of the residual (less than 0.4 ) is identified as the most probable malfunction candidate, among all possible candidates [1].

\subsection{Application 4: 320 MW steam turbo-generator with unbalance}

For the $320 \mathrm{MW}$ turbo-generator, represented in Fig. 16, coast-down transients before and after a balancing mass application were measured, so the additional vibrations due to an unbalance could be obtained.

Figure 14 represents the identification results: the identified position, modulus and phase are accurate for all speeds in the range 1400-2700 rpm (as in previous application), and the corresponding residual is low. In the low speed range and in the high speed range the position is always identified correctly, but the amplitude is affected by consistent errors and the phase by smaller errors.

Figure 17 shows that the single speed identification process has success in almost the entire speed range, but the best results are obtained in the speed range between 1500 and $3000 \mathrm{rpm}$. Position, amount and phase are identified with high accuracy. In particular the identification is successful at the machine rated speed. In the lower speed range the position is identified, but amount and phase become more uncertain.

\subsection{Application 5: 320 MW steam turbo-generator with local bow due to rub}

This application is related to a $320 \mathrm{MW}$ steam turbo-generator unit, see Fig. 18, which is one of the most common in Italian power plants. The rotor train is composed by two turbines, a HP-IP and a LP, and a generator connected by 


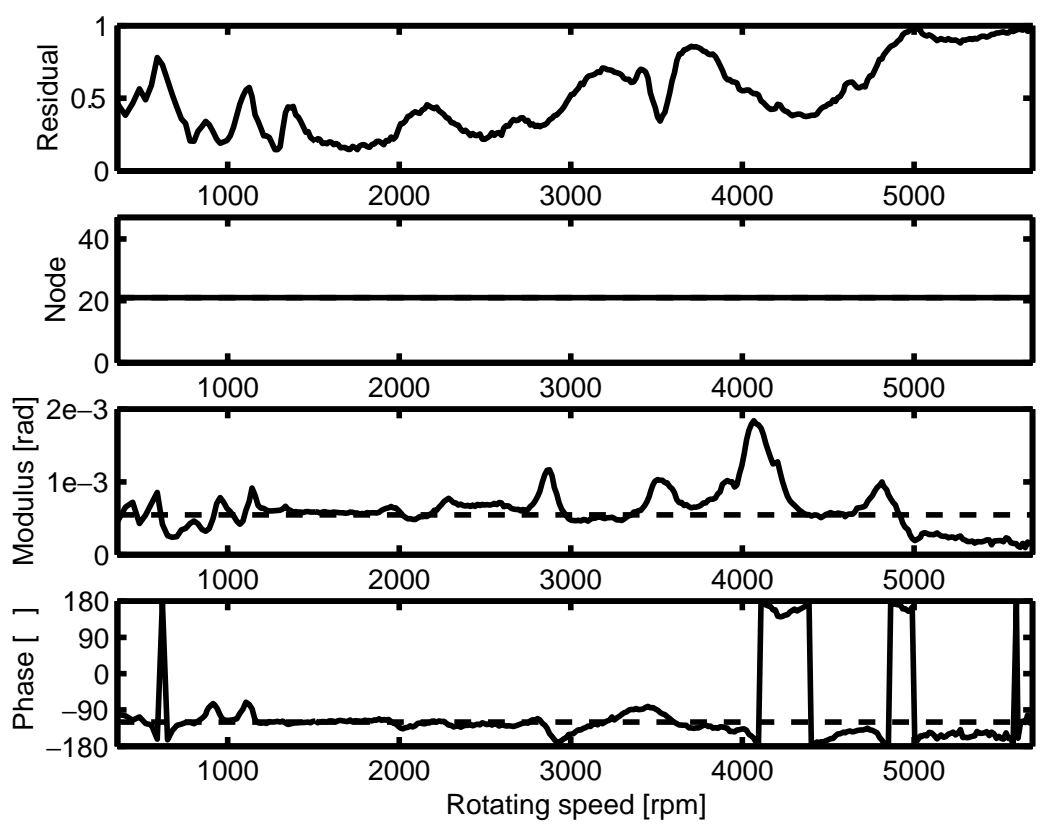

Fig. 14. Application 3: residual, node, modulus and phase identified as functions of the rotating speed.

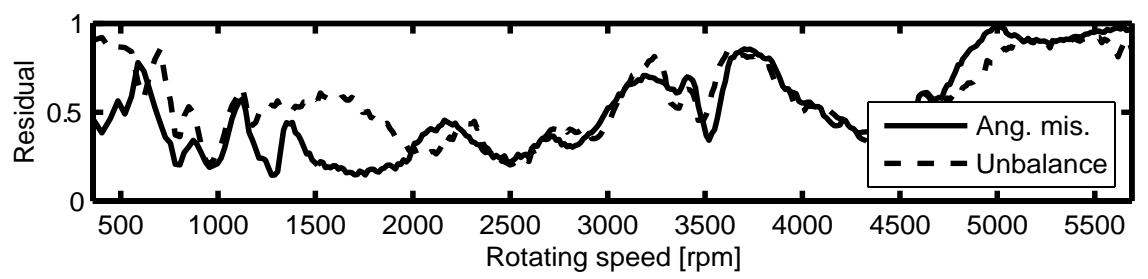

Fig. 15. Applications 3: comparison between the residuals of two different fault types.

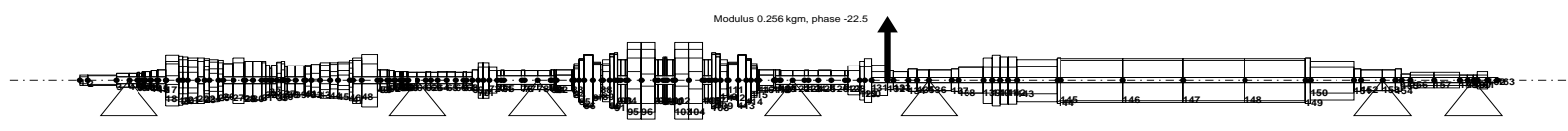

Fig. 16. Steam turbo-generator model.

rigid couplings, on seven oil film bearings operating at a rated speed of $3000 \mathrm{rpm}$. The rotor, which is about $28 \mathrm{~m}$ long and has a mass of about $126 \mathrm{t}$ ( $21 \mathrm{t}$ the HP-IP turbine, $49 \mathrm{t}$ the LP turbine and $56 \mathrm{t}$ the generator), has been modeled by 137 f.e. beams. The bearing stiffness and damping coefficients are defined for the rotating speeds equal to 1000,2000 and $3000 \mathrm{rpm}$, while the foundation is modeled by constant mass, spring and damper systems. The rotor model employed is the nominal one and no further tuning (model updating) has been done.

It is important to describe the application history in order to better comprehend the experimental data origin and the identification results. The machine started to show an abnormal vibrational behavior that indicated a possible rub in the LP turbine. The machine was then stopped. In order to reduce the vibrations, a second mode balancing (referred in the following as $1^{\text {st }}$ balancing) was performed by adding two balancing masses on two different balancing planes of LP turbine with a relative phase of $180^{\circ}$. After a two months operating period, while the abnormal behavior was still present, the machine was stopped again and another second mode balancing ( ${ }^{\text {nd }}$ balancing) was made with two balancing masses on the HP-IP turbine, on different balancing planes and with a relative phase of $180^{\circ}$. The abnormal behavior was still present and after an operating period of one month, the machine was stopped again and a further balancing ( $3^{\text {rd }}$ balancing) was made on one plane of the HP-IP turbine. Anyhow the problem was not solved 

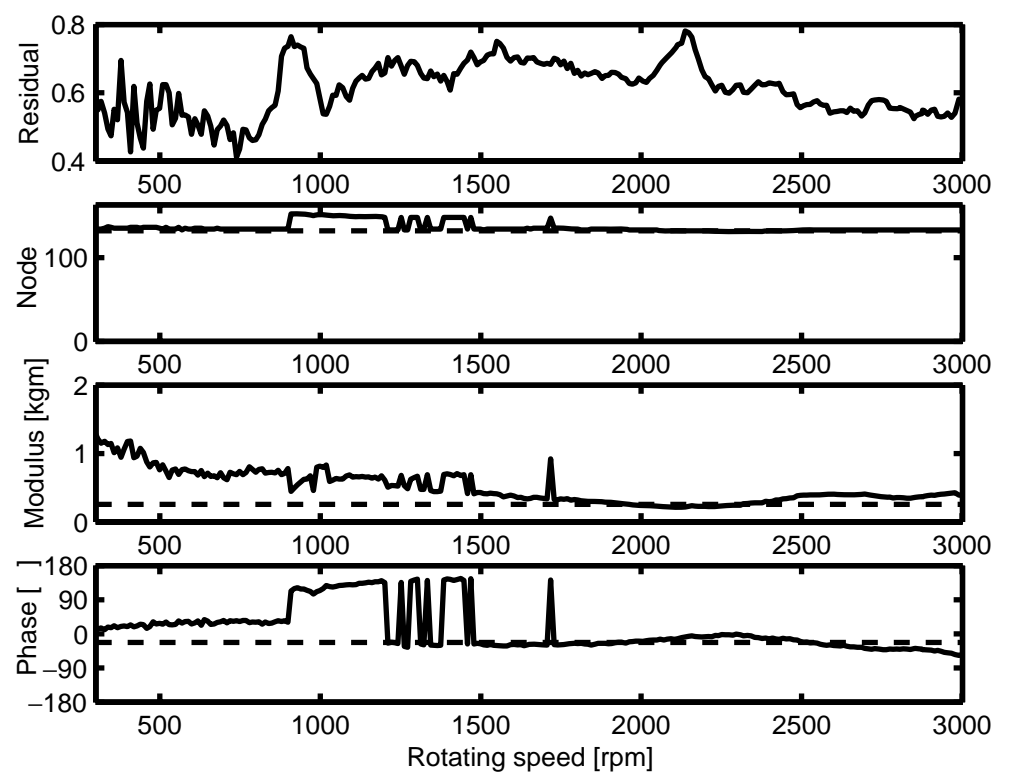

Fig. 17. Application 4: residual, node, modulus and phase identified as functions of the rotating speed.

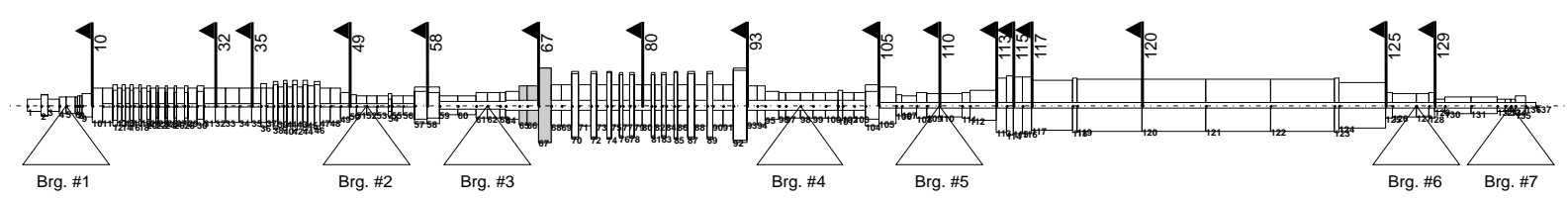

Fig. 18. $320 \mathrm{MW}$ steam turbo-generator model.

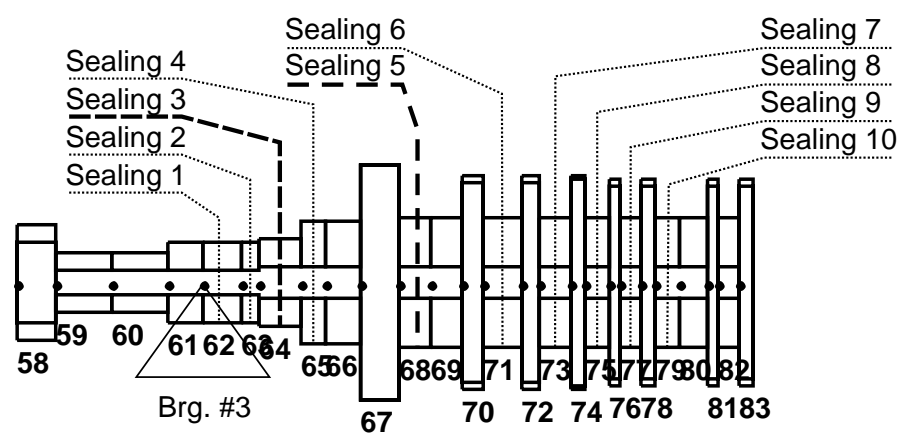

Fig. 19. Seal position close to LP last stage.

and finally the machine was stopped and a major maintenance made, which revealed that the seals on the LP turbine, close to bearing \#3, showed wear due to rub (Fig. 19 and shaded part of Fig. 18). All the positions and magnitudes of the balancing masses were defined without using a specific diagnostic procedure.

In this case, the identification procedure at rated speed is used for two different purposes: the first is to possibly identify the fault, the second is to define in a suitable way the balancing masses in order to reduce the vibration of the machine and avoid a major maintenance on it.

The additional vibrations due to the rub are determined by the difference of the vibrations at rated speed at the beginning of the coast-down for the $1^{\text {st }}$ balancing and a stored reference situation.

The single speed identification outputs at operating speed are shown in Fig. 20: the result can be considered good, 


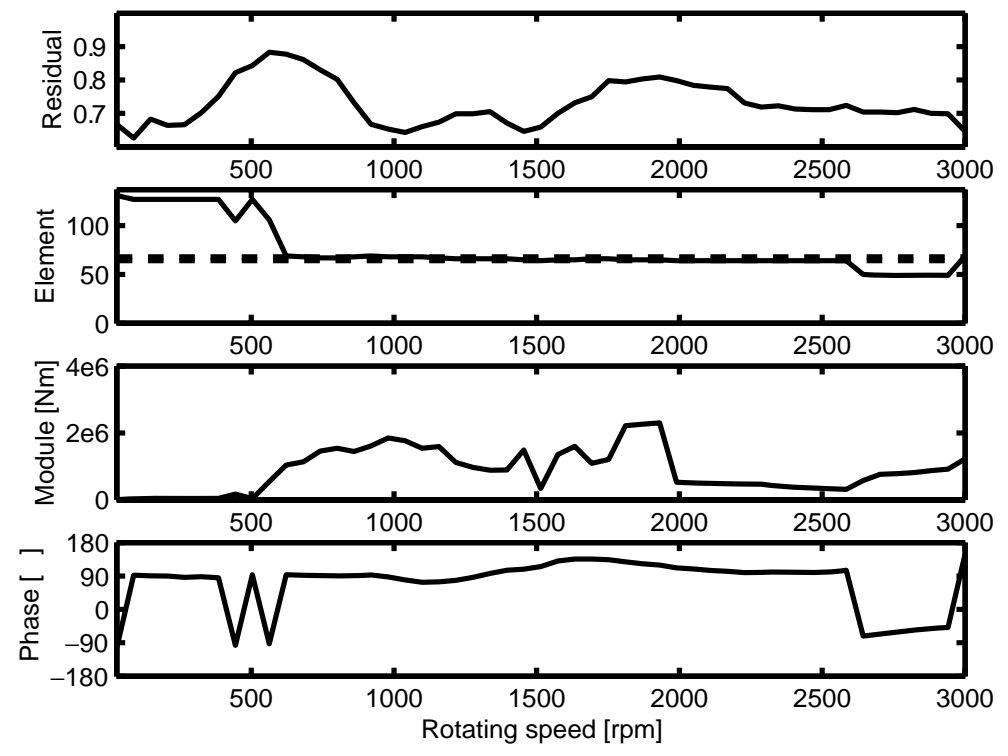

Fig. 20. Application 5: residual, node, modulus and phase identified as functions of the rotating speed.

discarding the speed range below $600 \mathrm{rpm}$, where the oil film bearing behavior becomes uncertain due to hydrostatic lubrication contribution, not considered in the model.

The bending moment which generates the local bow was changing, but also the rub condition was changing during the coast-down with changing vibration amplitudes. Further the bending moment values for a given local bow depend obviously also on diameter and length of the element in which the bow has developed. Therefore different bending moments will result from identification when the identified position changes from one element to another.

However the position, amount and phase are identified with good accuracy at rated speed, this is confirmed by the low residual. Comparison with other malfunctions, omitted for brevity, did not give any further indication about the nature of the malfunction.

Once the rub is indicated as the most probable cause of the abnormal dynamical behavior of the machine, two possibilities arise: the first is to stop the machine, perform a major not-scheduled maintenance and check the alignment and the clearances of all the seals; the second is to use some corrective balancing masses to reduce the vibrations, as was actually performed on the real machine. Anyhow, this correction is more effective (contrarily to what happened in the application history) if it is performed by means of a model based method.

Therefore the following procedure is used:

1) Define a possible force system, a corrective force system, that can determine a vibrational behavior similar to the additional vibrations at operating speed and that can be applied in opposite direction to the system.

2) Identify the position, the module and the phase of the corrective force system.

3) Verify that the additional vibrations at operating speed are reduced after the application of the corrective force system.

4) Verify that the corrective force system does not cause excessive vibration in transient speed condition (i.e. during the coast-down or the run-up) of the machine.

It is obvious that the simplest corrective force system is represented by some balancing masses. The problem now is to define the number and the position of the balancing masses that causes a vibrational behavior as close as possible to the additional vibrations and then apply these masses with opposite phase. The first attempt is made considering one balancing mass only.

Thus the problem of point 2 is to perform a fault identification, with a known fault type, at the operating speed, but with a further limitation: the position of the corrective force must coincide with the balancing planes of the machine (indicated by the vertical lines in Fig. 18). Therefore, in the algorithm presented in chapter 2, the procedure 


\section{Flexural dynamic deflection at $\mathbf{3 0 0 0}$ [rpm]}
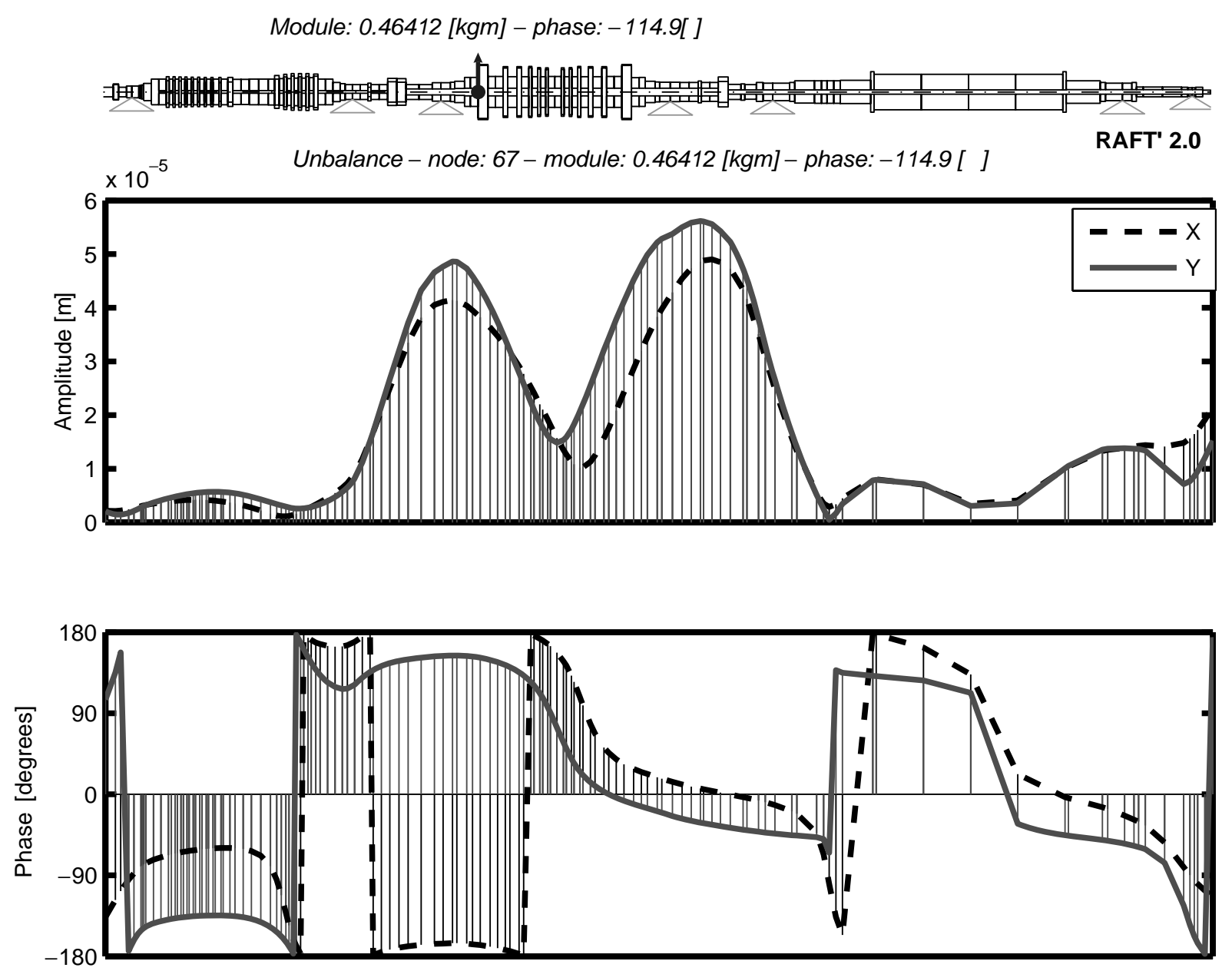

Fig. 21. Dynamic deflection at rated speed due to an unbalance on node 67.

is applied only to the nodes corresponding to the balancing planes, instead of applying it to all the nodes $n_{r}$. The results are shown in Table 1.

The residuals obtained in the nodes corresponding to the balancing planes are ranked in ascending order in the last column of Table 1 . The best positions of a corrective balancing mass are corresponding to the symmetric balancing planes close to the last stages of the LP turbine (nodes 67 and 93) and on the rigid coupling between the LP turbine and the generator (node 105). Note that the results in terms of residual and amplitude of the balancing mass are very similar for the LP extremity balancing planes, (the residuals are 0.75812 vs. 0.75845 , the modules 0.46412 vs. $0.46901 \mathrm{kgm})$ and the phases practically are $180^{\circ}$ shifted $\left(-114.9^{\circ}=245.1^{\circ} \mathrm{vs} .64 .163^{\circ}\right)$. This can be explained by considering that the LP turbine, at the rated speed of $3000 \mathrm{rpm}$ is very close to its $2 \mathrm{nd}$ flexural mode and that the dynamical deformation shape due to an unbalance in one of the two considered balancing planes, is practically symmetric, as shown in Fig. 21.

Four different possible corrective forces to correct the rub are evaluated:

- Case 1: balancing mass on plane 67, with amplitude $0.46412 \mathrm{kgm}$, phase $-114.9^{\circ}+180^{\circ}=65.1^{\circ}$ (corresponding to residual ranking \#1). 

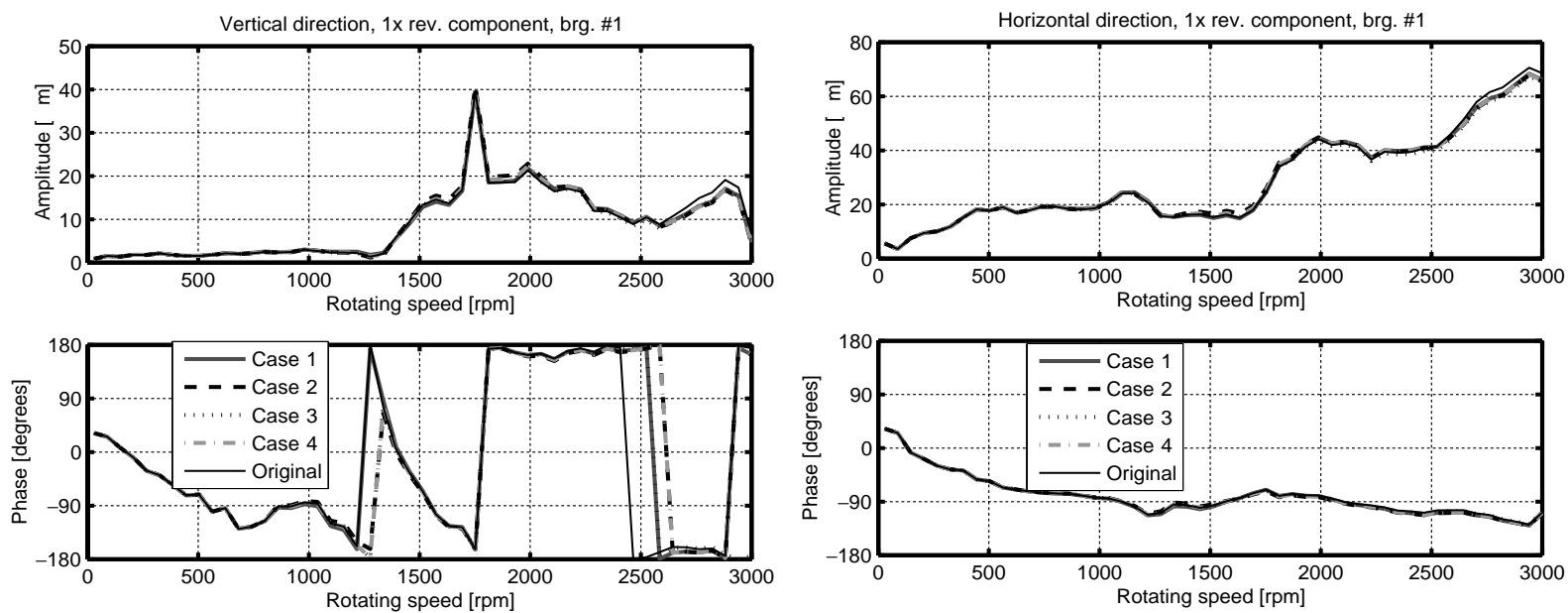

Fig. 22. Frequency response in bearing \#1.
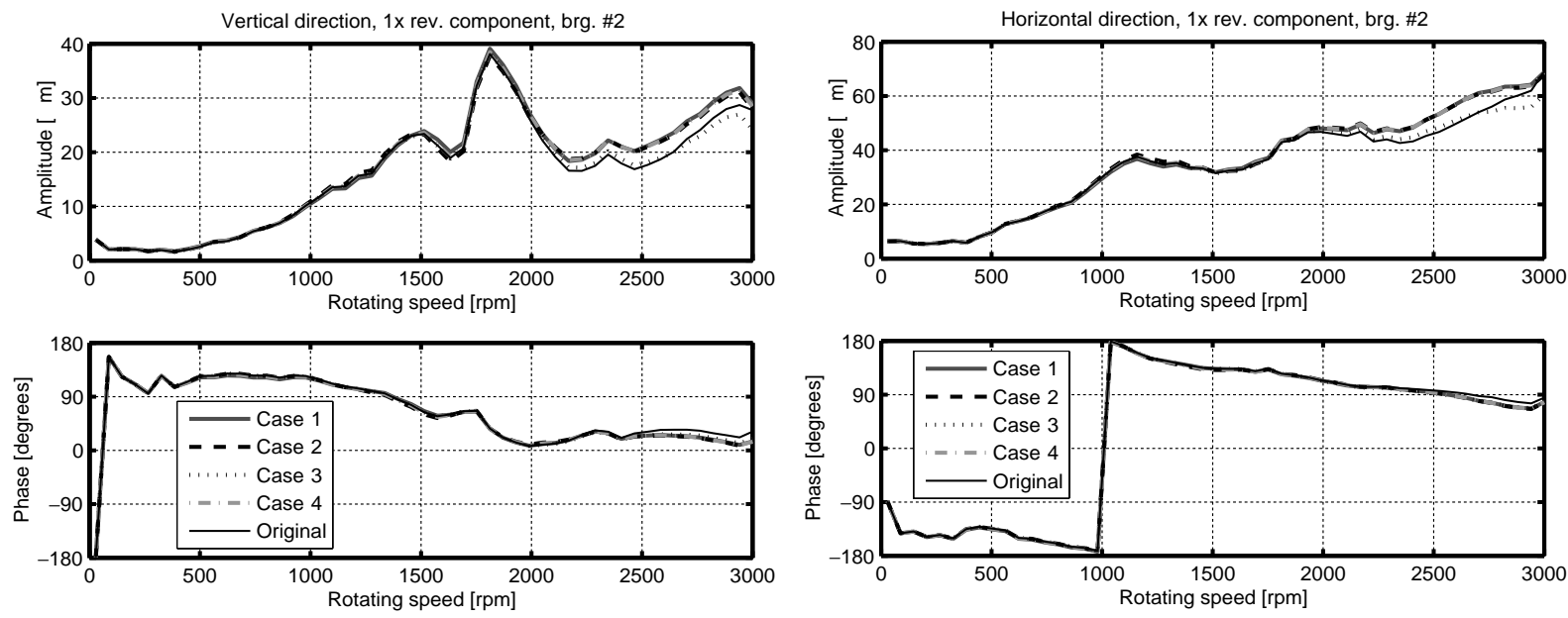

Fig. 23. Frequency response in bearing \#2.

- Case 2: balancing mass on plane 93, with amplitude $0.46901 \mathrm{kgm}$, phase $64.136^{\circ}-180^{\circ}=-115.864^{\circ}$ (corresponding to residual ranking \#2).

- Case 3: balancing mass on plane 105, with amplitude $0.34844 \mathrm{kgm}$, phase $51.74^{\circ}-180^{\circ}=-128.26^{\circ}$ (corresponding to residual ranking \#3); this balancing plane on the coupling is much easier to access than the other balancing planes.

- Case 4: a $2^{\text {nd }}$ mode balancing can be used applying two balancing masses on the LP extremity balancing planes: a first mass on plane 67, with amplitude $0.233 \mathrm{kgm}$, phase $-114.9^{\circ}+180^{\circ}=65.1^{\circ}$ and the second on plane 93 , with amplitude $0.233 \mathrm{kgm}$, phase $64.136^{\circ}-180^{\circ}=-115.864^{\circ}$. The amplitudes of the corrective masses have been assumed half of the average balancing mass of cases 1 and 2 .

The results are evaluated by simulating the dynamic behavior of the machine due to each corrective force system, over the entire speed range $0-3000 \mathrm{rpm}$, and subtracting these forced vibrations from the additional vibrations. This way, under the hypothesis of linearity of the system, the resulting vibrations represent the machine behavior in presence of the fault and its correction.

Since the machine experienced rub at operating speed, the first goal is to reduce the vibrations at rated speed with the corrective force system; anyhow it is necessary to check that the corrective force system allows the crossing of the 

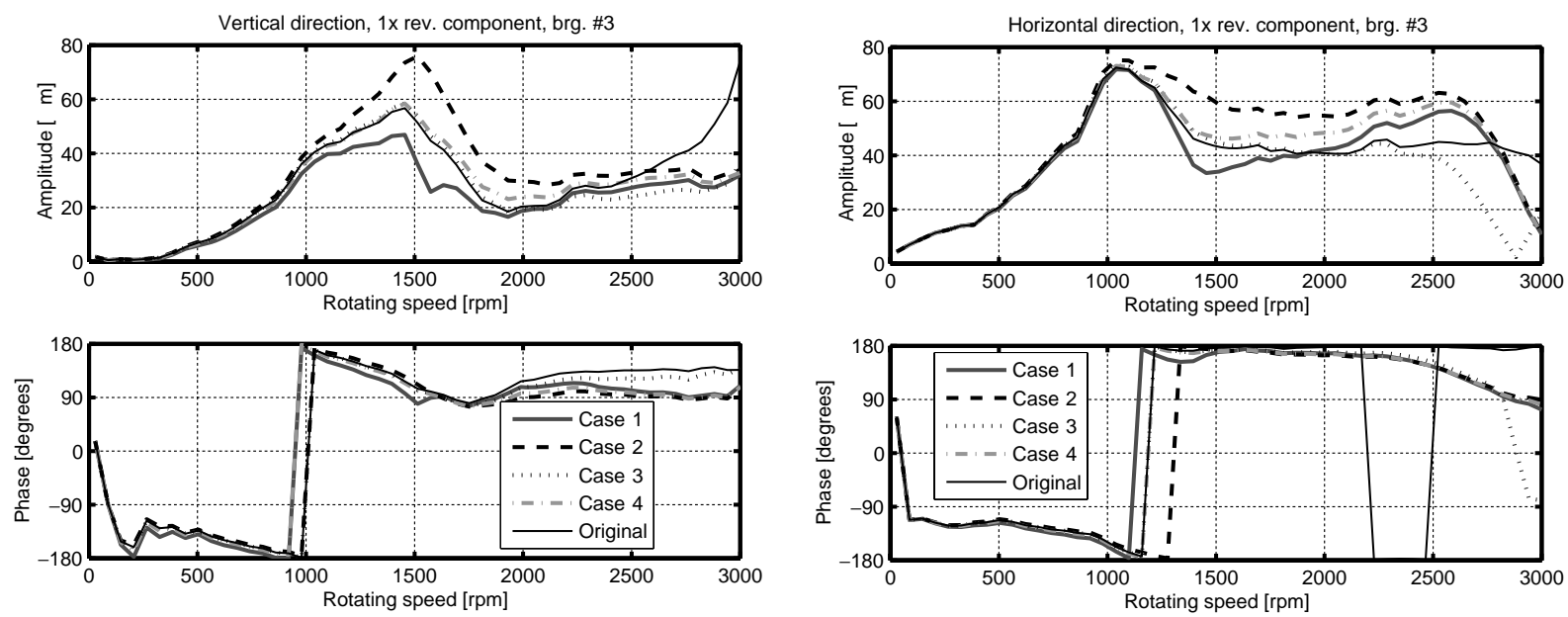

Fig. 24. Frequency response in bearing \#3.
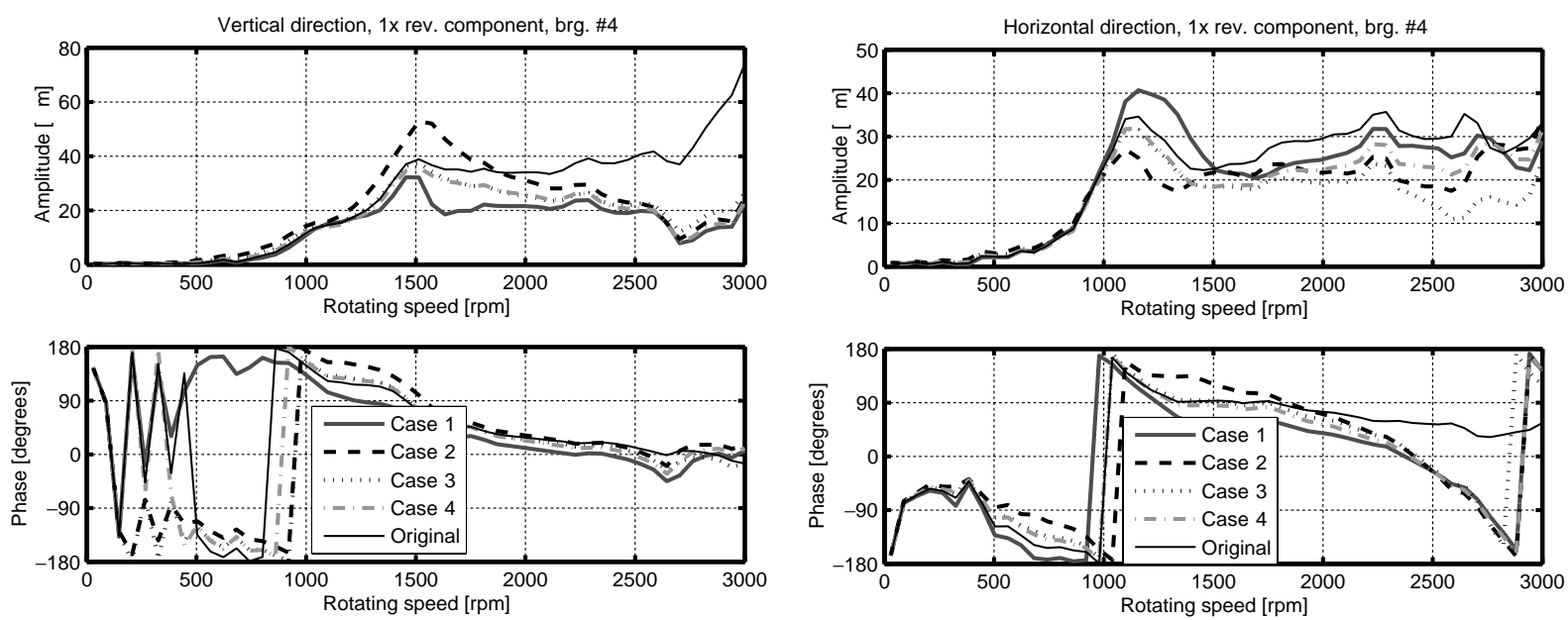

Fig. 25. Frequency response in bearing \#4.

machine critical speeds, during the run-up and coast-down transients, without inducing limit exceeding vibrations. The results are discussed hereafter.

\subsubsection{Effects of the corrective forces at the operating speed}

The results obtained in the different cases at the operating speed are reported in Table 2 for the vibration amplitude.

As a general comment, the corrective forces applied on the LP balancing planes (cases 1, 2 and 4) determine higher reduction of the vibrations on the LP bearings than case 3, except for the horizontal direction of bearing \#4. The effect in all the cases on the HP-IP turbine is comparable and not very important. Instead, on the generator, case 4 determines a strong amplification of the vibration of bearings \#5 and \#6, while the other cases amplifies the vibrations of exciter bearing \#7 in horizontal direction. Anyhow, the effect on the generator part has to be evaluated not only in terms of relative variation, but also in absolute terms. If this criterion is used, the vibration amplitude is acceptable.

\subsubsection{Effects of the corrective forces during the speed transient}

For the sake of brevity and to have a rapid comparison, the diagrams are superposed for all the cases and for the original (additional) vibrations. The differences between the responses before and after the corrective force are 

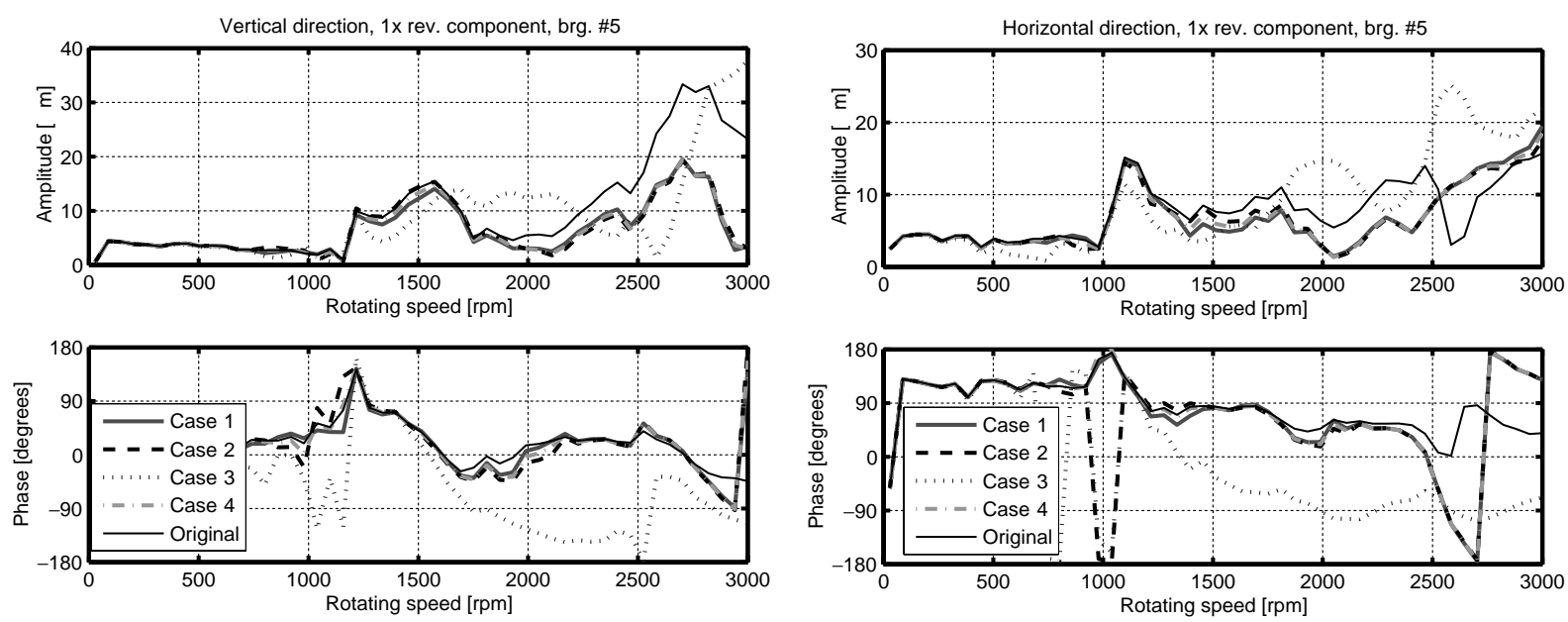

Fig. 26. Frequency response in bearing \#5.
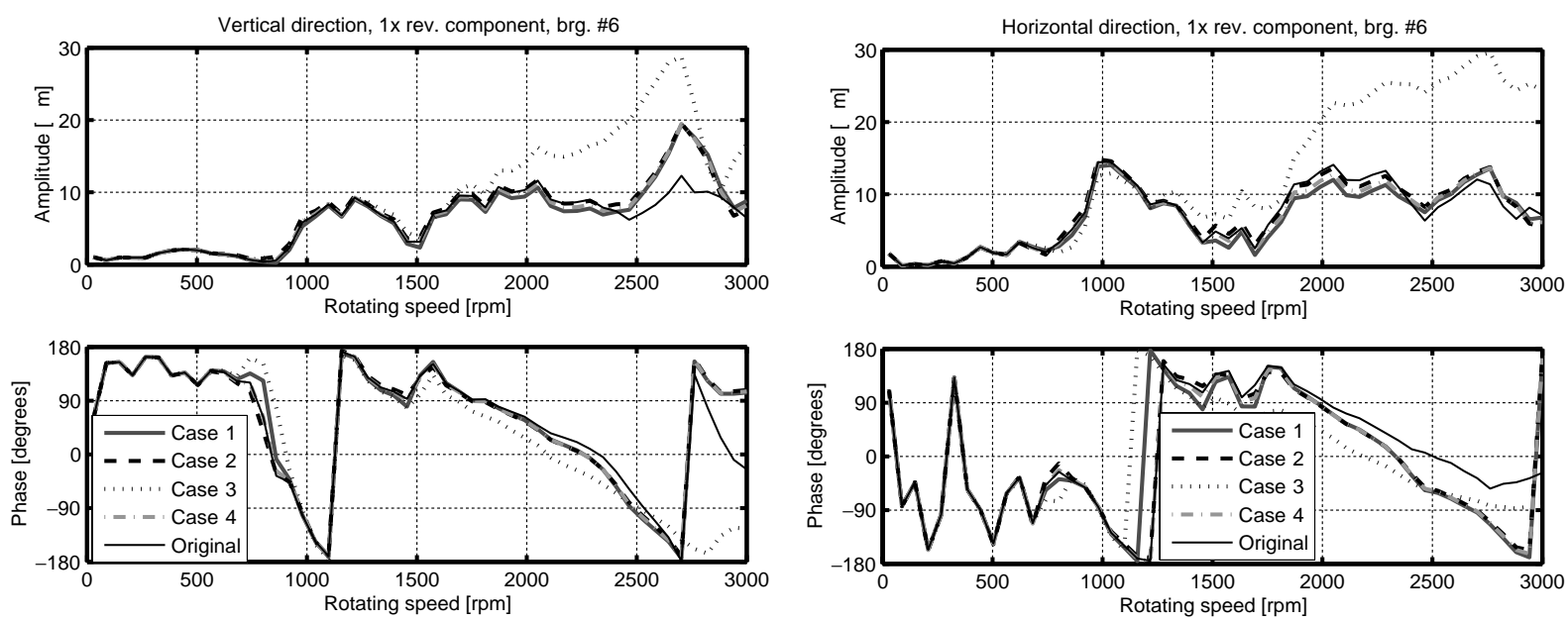

Fig. 27. Frequency response in bearing \#6.

vanishing small for the HP-IP turbine (Figs 22 and 23), and the crossing of the critical speed on the HP-IP turbine is practically uninfluenced by the corrective forces.

The responses for the LP bearings, shown in Figs 24 and 25, exhibit that case 2 causes higher vibration levels than the other cases, before reaching the operating speed, except in the horizontal direction of bearing \#4. Case 1 causes instead an antithetic situation during the speed transient with lower dynamic amplifications crossing the critical speeds except in the horizontal direction of bearing \#4. The results obtained with the corrective force of case 3 are comparable to case 4 and both are in between cases 1 and 2 results.

On the generator bearings, whose responses are shown in Figs 26 and 27, the corrective force of case 3 causes generally higher vibration levels during the speed transient than the other cases, which are practically equivalent. Anyhow the vibration levels are rather small.

Finally, on the exciter bearing, Fig. 28, the response of corrective force of case 3 has generally lower amplitude than other cases.

\subsubsection{Results}

On the basis of the previous analysis, the corrective forces of case 1, that corresponds to the balancing plane close to the seals interested by rub, give the best results in reducing the vibrations of the LP turbine at operating speed and 
Table 1

Corrective force identification

\begin{tabular}{ccclc}
\hline $\begin{array}{c}\text { Node of the } \\
\text { balancing plane }\end{array}$ & $\begin{array}{c}\text { Module } \\
{[\mathrm{kgm}]}\end{array}$ & $\begin{array}{c}\text { Phase }^{(1)} \\
\text { [degrees] }\end{array}$ & Residual & $\begin{array}{r}\text { Residual } \\
\text { ranking }\end{array}$ \\
\hline 10 & 0.92576 & -142.91 & 0.77781 & 4 \\
32 & 0.4194 & -158.66 & 0.96537 & 10 \\
35 & 0.12261 & 162.4 & 0.99774 & 16 \\
49 & 0.83719 & 25.61 & 0.88501 & 5 \\
58 & 0.26567 & -89.737 & 0.9423 & 8 \\
67 & 0.46412 & -114.9 & 0.75812 & 1 \\
80 & 0.99568 & -131.58 & 0.9148 & 7 \\
93 & 0.46901 & 64.163 & 0.75845 & 2 \\
105 & 0.34844 & 51.74 & 0.76226 & 3 \\
110 & 0.27886 & 42.059 & 0.90421 & 6 \\
113 & 0.16963 & 40.241 & 0.9776 & 13 \\
115 & 0.11895 & 42.748 & 0.98983 & 14 \\
117 & 0.06888 & 50.862 & 0.99693 & 15 \\
120 & 0.50059 & -156.95 & 0.96665 & 11 \\
125 & 0.31433 & 21.628 & 0.95282 & 9 \\
129 & 0.092477 & 20.4 & 0.96931 & 12 \\
\hline
\end{tabular}

(1) Note that this phase is that of the force system that match in least square sense the vibration at operating speed of the system. The corrective force has opposite phase.
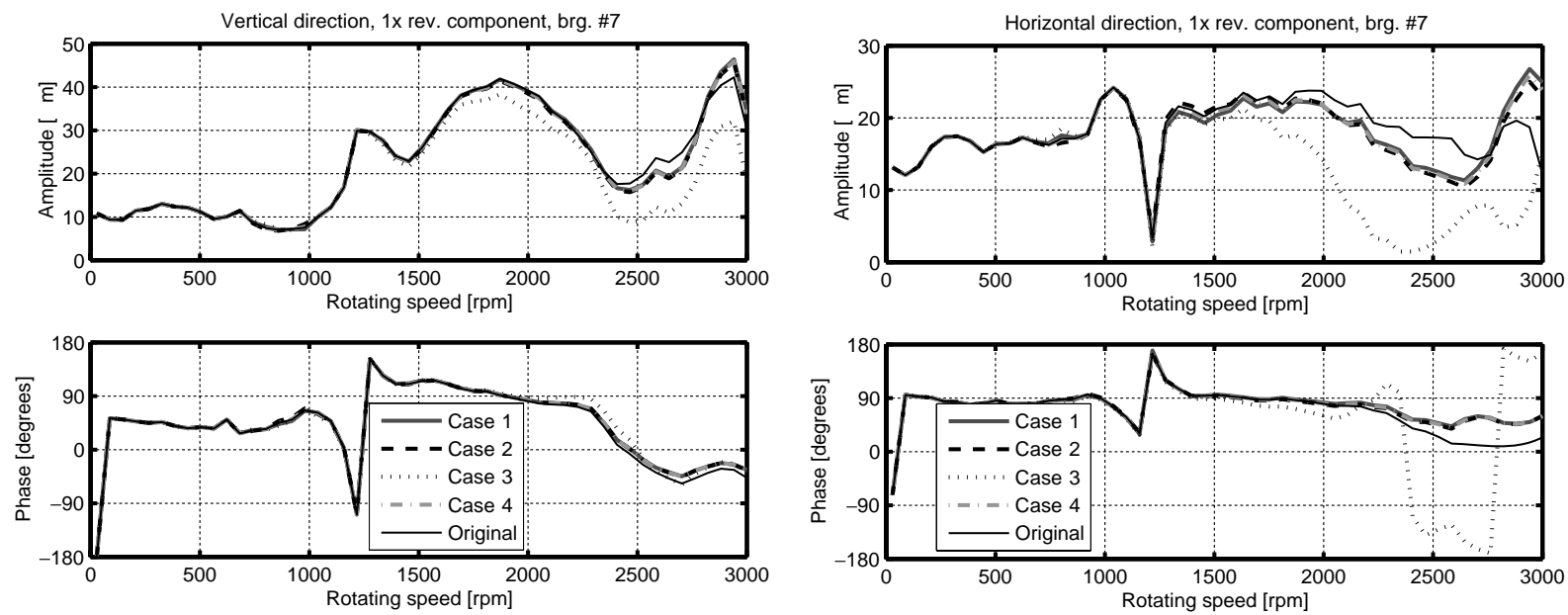

Fig. 28. Frequency response in bearing \#7.

in avoiding excessive high vibrations when crossing the critical speeds. Anyhow, since the access to this balancing plane could require a longer outage of the machine, a sub-optimal solution, that guarantees in any case a consistent reduction of the vibration amplitudes at operating speed, is case 3, whose balancing plane is easily accessible.

In the considered application, the identification at operating speed allows to define a corrective force system, consisting of balancing masses placed on balancing planes, which reduces the vibrations of the faulty machine at rated speed. The behavior of the machine during coast-down transients has also to be checked and this allows to select the optimum corrective force system.

\section{Conclusions}

Single speed model based identification of faults or malfunctions can be successful, provided that the system model is linear and time invariant. 
Table 2

Results of application of corrective forces at operating speed

\begin{tabular}{|c|c|c|c|c|c|c|c|c|c|}
\hline & \multirow[t]{2}{*}{ Additional } & \multicolumn{2}{|c|}{ Case 1} & \multicolumn{2}{|c|}{ Case 2} & \multicolumn{2}{|c|}{ Case 3} & \multicolumn{2}{|c|}{ Case 4} \\
\hline & & $\begin{array}{c}\text { After } \\
\text { correction }\end{array}$ & Variation & $\begin{array}{c}\text { After } \\
\text { correction }\end{array}$ & Variation & $\begin{array}{c}\text { After } \\
\text { correction }\end{array}$ & Variation & $\begin{array}{c}\text { After } \\
\text { correction }\end{array}$ & Variation \\
\hline Brg \#1 Vert & 6.56 & 4.67 & $-28.80 \%$ & 4.28 & $-34.79 \%$ & 4.45 & $-32.18 \%$ & 4.48 & $-31.74 \%$ \\
\hline Brg \#1 Hor & 68.52 & 66.06 & $-3.60 \%$ & 65.47 & $-4.45 \%$ & 65.74 & $-4.06 \%$ & 65.77 & $-4.02 \%$ \\
\hline Brg \#2 Vert & 27.73 & 28.96 & $4.40 \%$ & 28.01 & $0.96 \%$ & 24.18 & $-12.82 \%$ & 28.48 & $2.68 \%$ \\
\hline Brg \#2 Hor & 69.07 & 68.78 & $-0.42 \%$ & 67.85 & $-1.76 \%$ & 60.47 & $-12.45 \%$ & 68.32 & $-1.09 \%$ \\
\hline Brg \#3 Vert & 74.18 & 31.99 & $-56.87 \%$ & 33.80 & $-54.44 \%$ & 36.40 & $-50.92 \%$ & 32.90 & $-55.64 \%$ \\
\hline Brg \#3 Hor & 36.69 & 10.80 & $-70.55 \%$ & 11.26 & $-69.31 \%$ & 18.46 & $-49.69 \%$ & 10.90 & $-70.30 \%$ \\
\hline Brg \#4 Vert & 74.05 & 22.48 & $-69.64 \%$ & 24.59 & $-66.80 \%$ & 26.59 & $-64.09 \%$ & 23.52 & $-68.23 \%$ \\
\hline Brg \#4 Hor & 33.12 & 28.96 & $-12.56 \%$ & 33.90 & $2.37 \%$ & 24.62 & $-25.65 \%$ & 31.38 & $-5.24 \%$ \\
\hline Brg \#5 Vert & 23.20 & 3.40 & $-85.34 \%$ & 3.00 & $-87.07 \%$ & 37.69 & $62.43 \%$ & 2.98 & $-87.15 \%$ \\
\hline Brg \#5 Hor & 15.70 & 19.49 & $24.14 \%$ & 17.51 & $11.55 \%$ & 21.96 & $39.85 \%$ & 18.49 & $17.74 \%$ \\
\hline Brg \#6 Vert & 6.46 & 8.81 & $36.45 \%$ & 7.58 & $17.42 \%$ & 16.71 & $158.73 \%$ & 8.18 & $26.67 \%$ \\
\hline Brg \#6 Hor & 7.11 & 6.79 & $-4.45 \%$ & 5.55 & $-21.95 \%$ & 24.38 & $242.94 \%$ & 6.14 & $-13.67 \%$ \\
\hline Brg \#7 Vert & 30.27 & 34.22 & $13.07 \%$ & 33.35 & $10.17 \%$ & 18.23 & $-39.75 \%$ & 33.78 & $11.61 \%$ \\
\hline Brg \#7 Hor & 12.19 & 24.90 & $104.22 \%$ & 23.00 & $88.38 \%$ & 15.36 & $25.98 \%$ & 23.93 & $96.20 \%$ \\
\hline
\end{tabular}

Conditions close to resonances should be avoided for succeeding in the identification procedure. This fact is probably due to inaccuracies of the models, which are unable to predict the vibrations of complex systems for such conditions where amplitudes and phases are rapidly changing.

The discrimination between different malfunctions which generate similar symptoms (e.g. faults that generate only $1 \mathrm{xrev}$. vibration components) seems to be impossible with single speed identification: it is possible only considering different rotating speeds.

Despite these drawbacks of single speed identification, it seems to be possible to use this approach also in industrial applications, checking accurately the obtained results and being aware of its limitations. Balancing procedure results could be predicted by identifying the unbalance in correspondence of each one of the available balancing planes: same unbalance placed in opposite angular position $\left(180^{\circ}\right)$ in the balancing planes should be able to reduce consistently the measured vibrations, independently of the real type of malfunction which caused the measured additional vibrations. Therefore the behaviour of a rotor with a coupling misalignment or a bow could be corrected by suitable balancing masses.

Single speed identification could suggest position and amount of suspected fault, but this identification should be validated by the data collected during coast-down transients.

\section{Acknowledgements}

The financial support of MIUR (PRIN project "Diagnostics of rotor systems in industrial plants") is gratefully acknowledged.

\section{References}

[1] N. Bachschmid and P. Pennacchi, Accuracy of fault detection in real rotating machinery using model based diagnostic techniques, JSME International Journal Series C 46(3) (2003), 1026-1034.

[2] N. Bachschmid, P. Pennacchi and A. Vania, Identification of Multiple Faults in Rotor Systems, Journal of Sound and Vibration 254(2) (2002), 327-366.

[3] R. Markert, R. Platz and M. Siedler, Model Based Fault Identification in Rotor Systems by Least Squares Fitting, ISROMAC-8 Conference, Honolulu, Hawaii, 901-915.

[4] P. Pennacchi, N. Bachschmid, A. Vania, G.A. Zanetta and L. Gregori, Use of Modal Representation for the Supporting Structure in Model Based Fault Identification of Large Rotating Machinery: Part 1 - Theoretical Remarks, Mechanical Systems and Signal Processing 20(3) (2006), 662-681.

[5] R. Platz, R. Markert and M. Seidler, Validation of online diagnostics of malfunctions in rotor systems, IMechE paper C576-025, 7th Int. Conf. on Vibrations in Rotating Machinery, University of Nottingham, 581-590. 

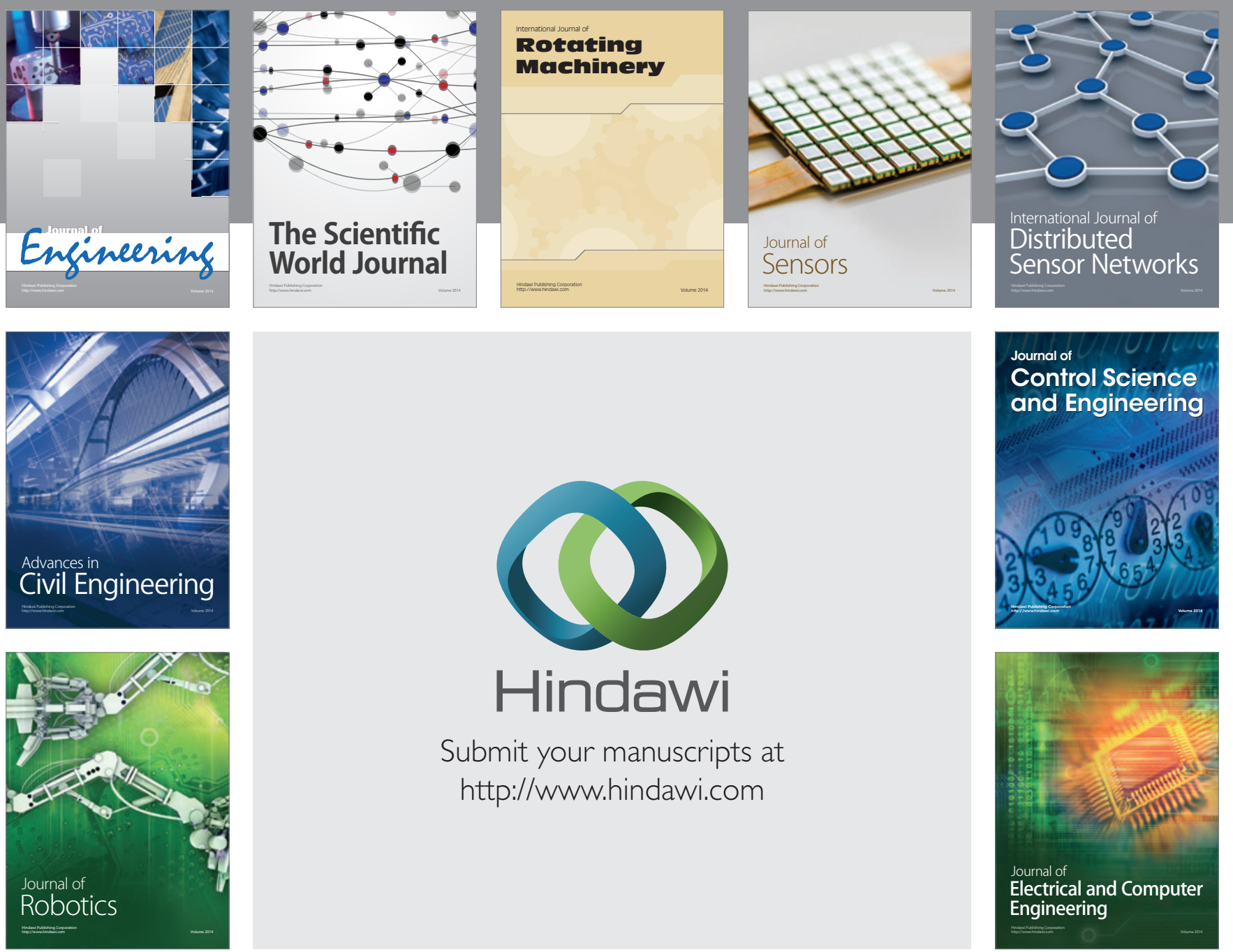

Submit your manuscripts at

http://www.hindawi.com
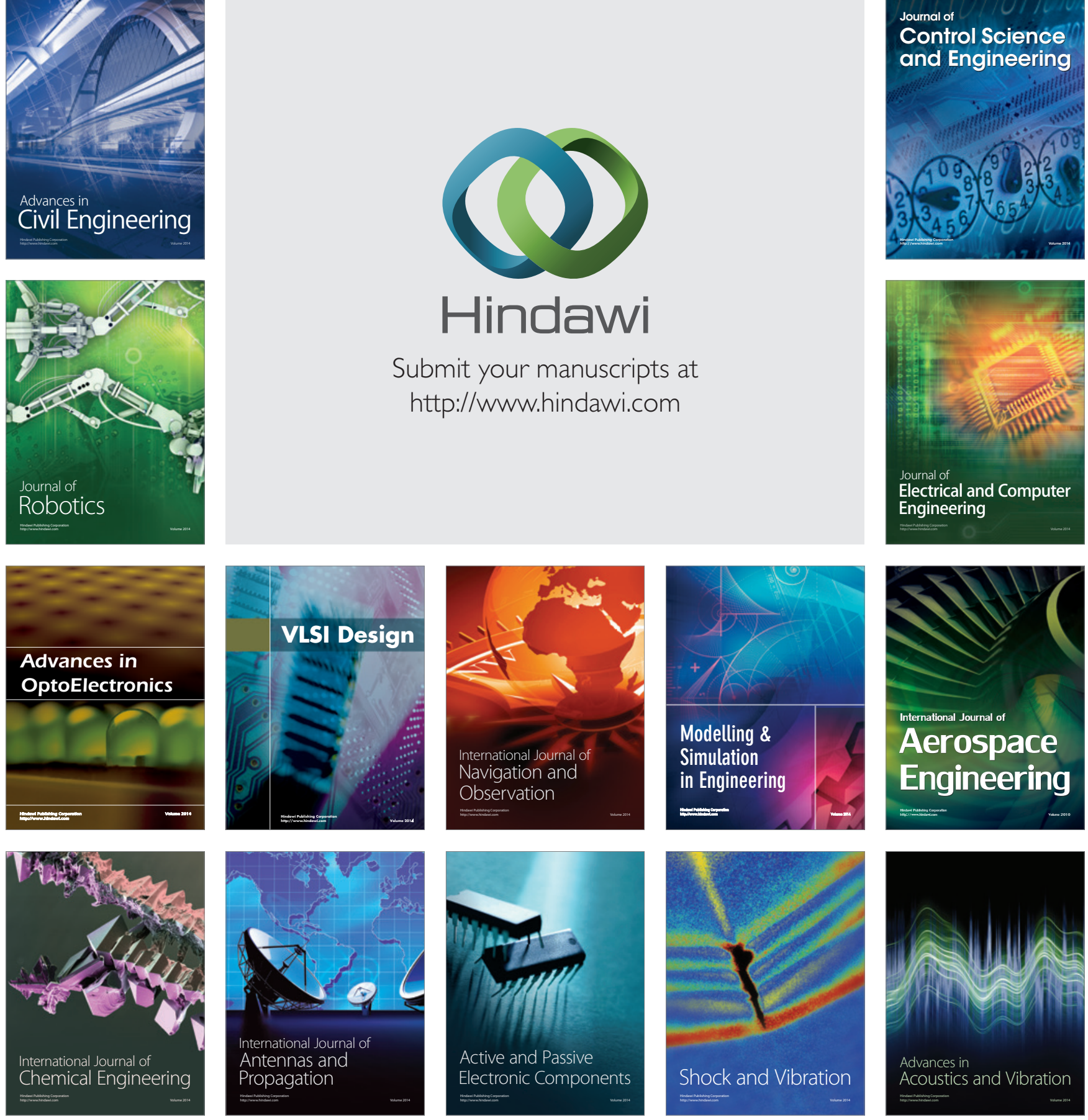